24h after knockdown: THRa expression

Predicted band size: $55 \mathrm{kDa}, \beta$-Actin $42 \mathrm{kDa}$

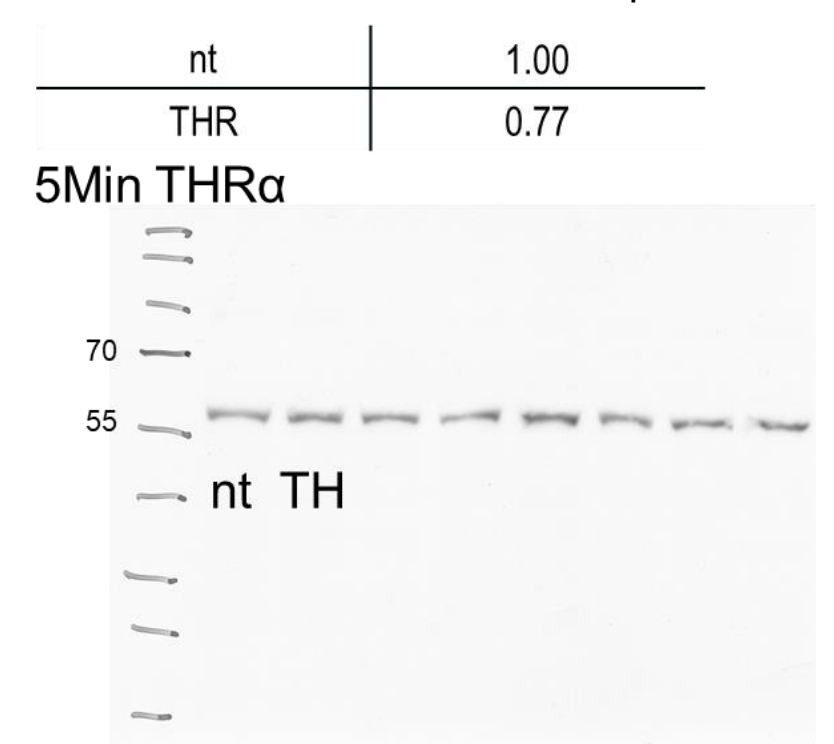

$1 \sec \beta$-Actin

70 - -

55

40 nt $\mathrm{TH}$

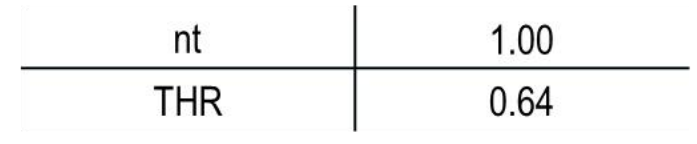

$1 \mathrm{Min}$ THTa

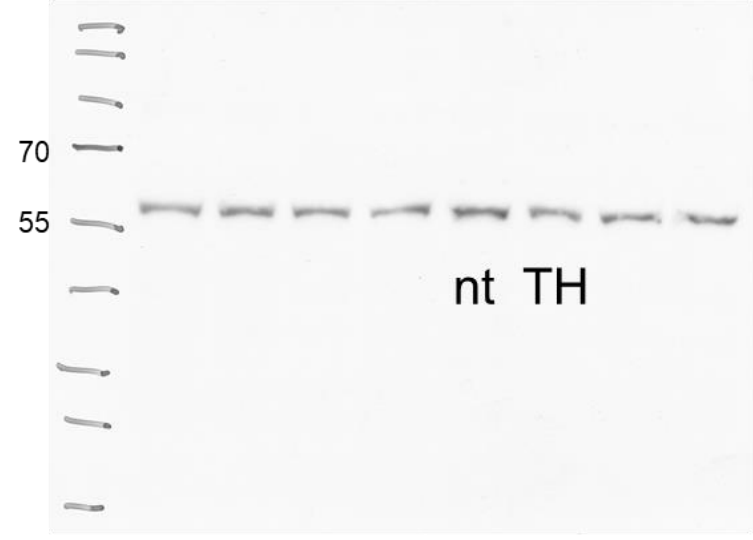

$1 \sec \beta$-Actin

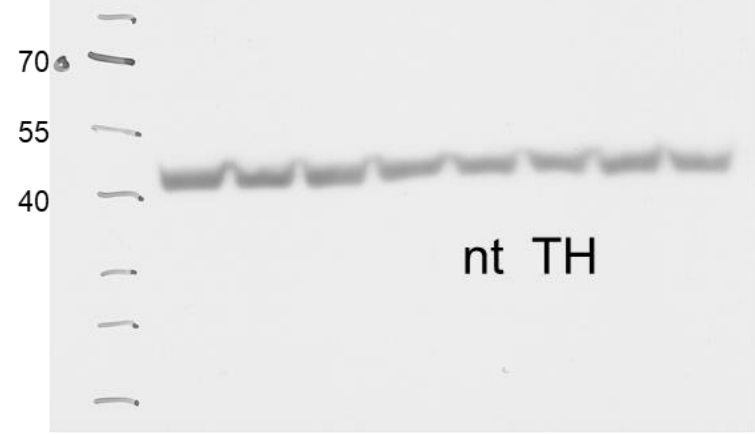

\begin{tabular}{c|c|}
\hline $\mathrm{nt}$ & 1.00 \\
\hline THR & 0.88 \\
\hline
\end{tabular}

1Min THRa

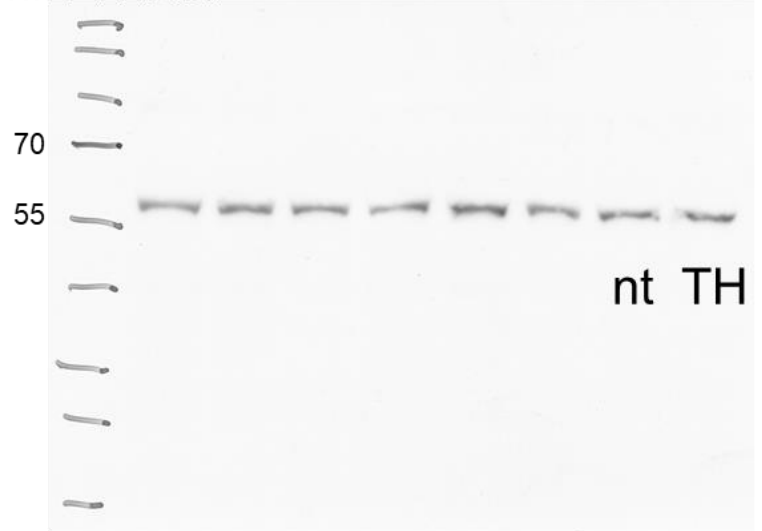

3sec $\beta$-Actin

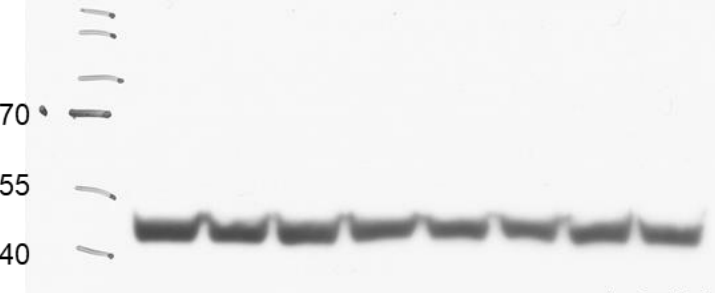

nt TH

\begin{tabular}{c|c|} 
nt & 1.00 \\
\hline THR & 0.82
\end{tabular}

$30 \mathrm{sec}$ THRa

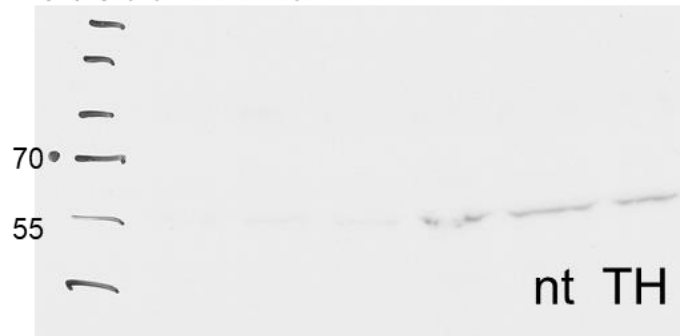

-

3sec $\beta$-Actin

$70 \ldots$

55

40

nt $\mathrm{TH}$ 
48h after knockdown: THRa expression

Predicted band size: $55 \mathrm{kDa}, \beta$-Actin $42 \mathrm{kDa}$

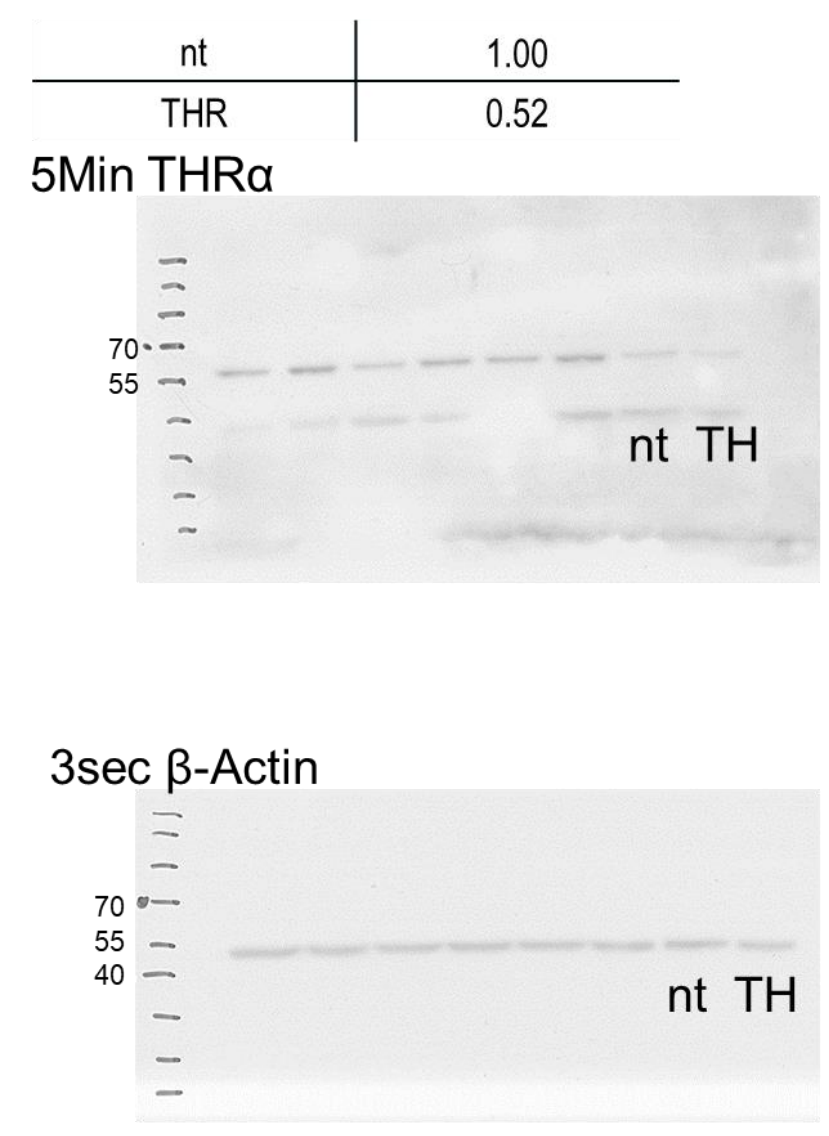

\begin{tabular}{|c|l|}
\hline nt & 1.00 \\
\hline THR & 0.10 \\
\hline 5Min THRa
\end{tabular}

5 Min THRa

\begin{tabular}{|c|l|}
\hline nt & 1.00 \\
\hline THR & 0.42 \\
\hline 1Min THRa
\end{tabular}

1Min THRa

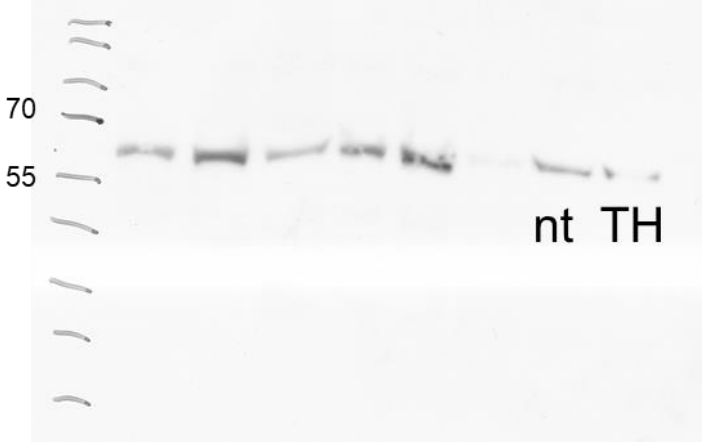

$1 \sec \beta$-Actin

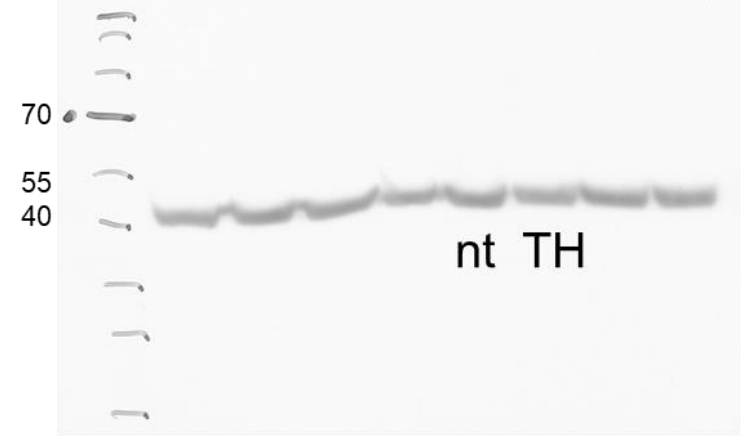

\section{3sec $\beta$-Actin}

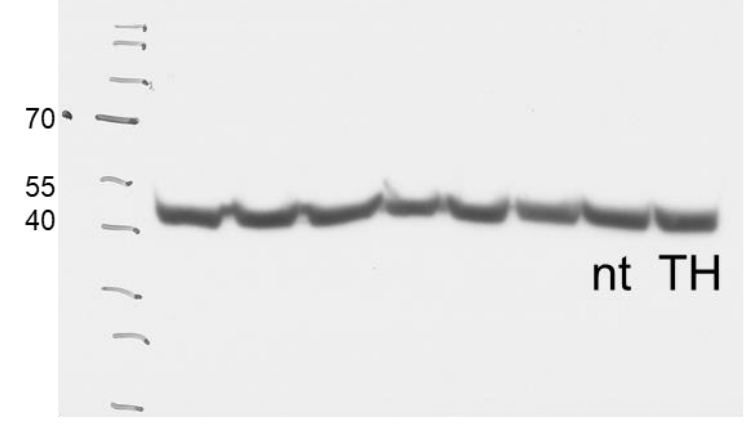

\begin{tabular}{c|l}
\hline $\mathrm{nt}$ & 1.00 \\
\hline THR & 0.39
\end{tabular}

30 sec THRa

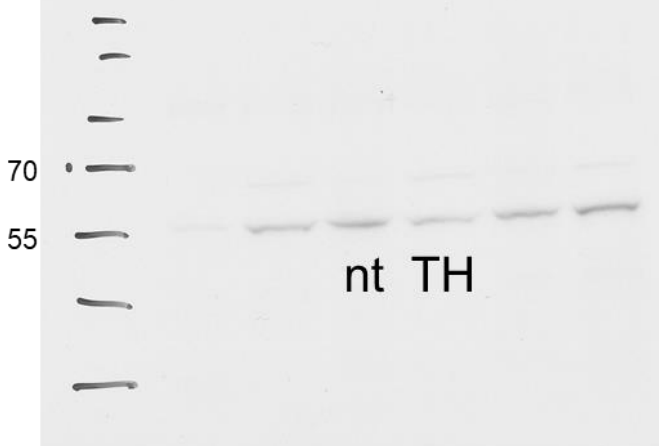

3sec $\beta$-Actin

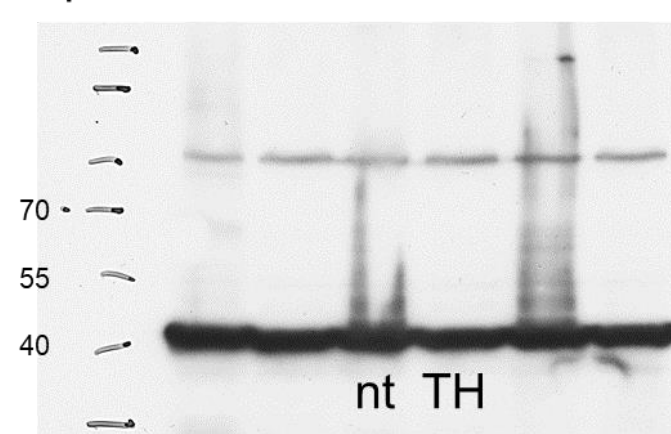




\section{8h after knockdown: THR $\beta$ expression}

Predicted band size: Isoform Beta 153 kDa, Isoform Beta 254 kDa, $\beta$-Actin 42 kDa
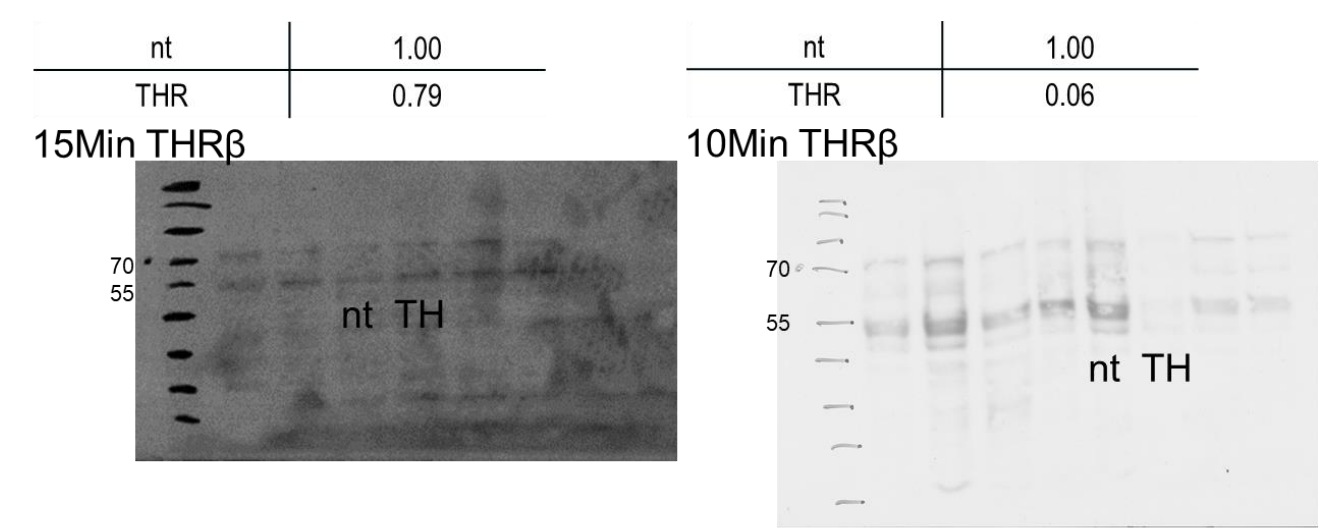

3sec $\beta$-Actin

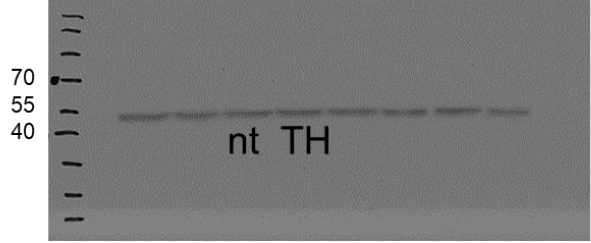

3sec $\beta$-Actin

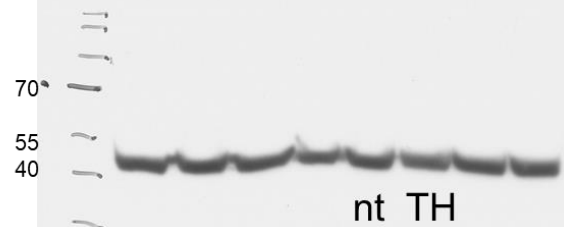

nt TH

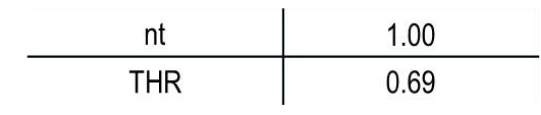

10Min THR $\beta$

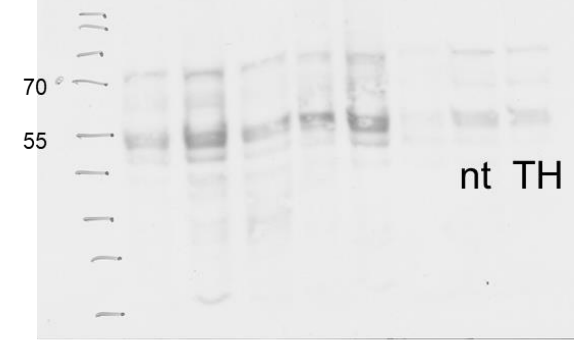

3sec $\beta$-Actin

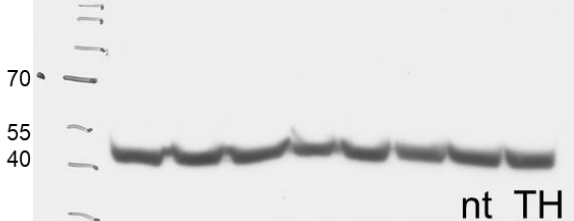

nt TH

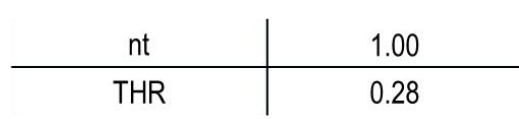

10Min THRß

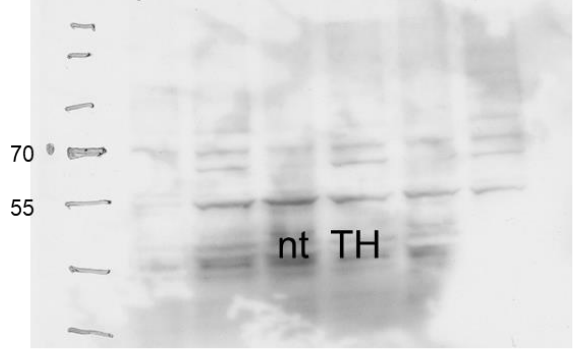

3sec $\beta$-Actin

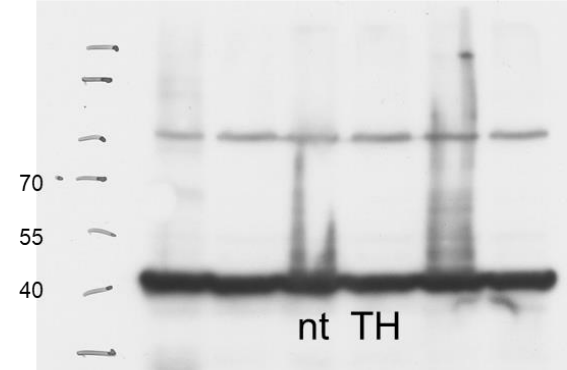

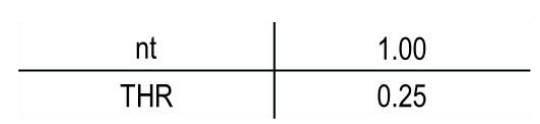

10Min THRß

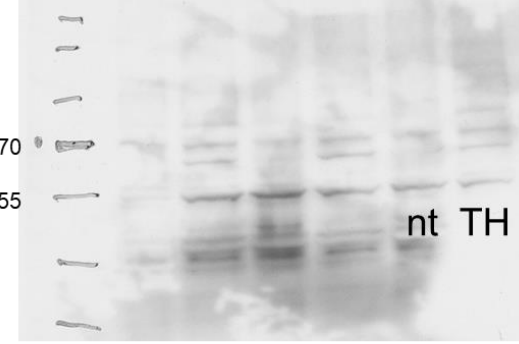

$3 \sec \beta$-Actin

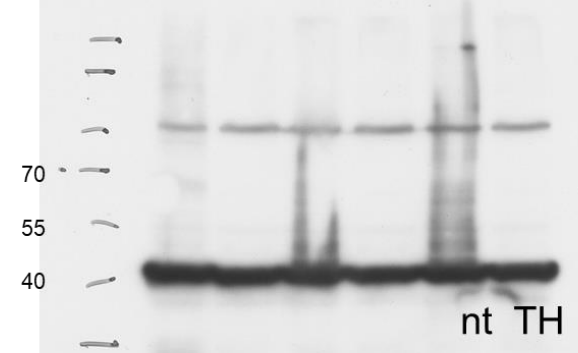


24h after transfection with non-targeting siRNA: T4 induced CD36 expression

Predicted band size: $53 \mathrm{kDa}, \beta$-Actin $42 \mathrm{kDa}$

\begin{tabular}{c|c}
0 & 1.00 \\
\hline $\mathrm{T} 4$ & 1.63
\end{tabular}

$30 \sec$ CD36

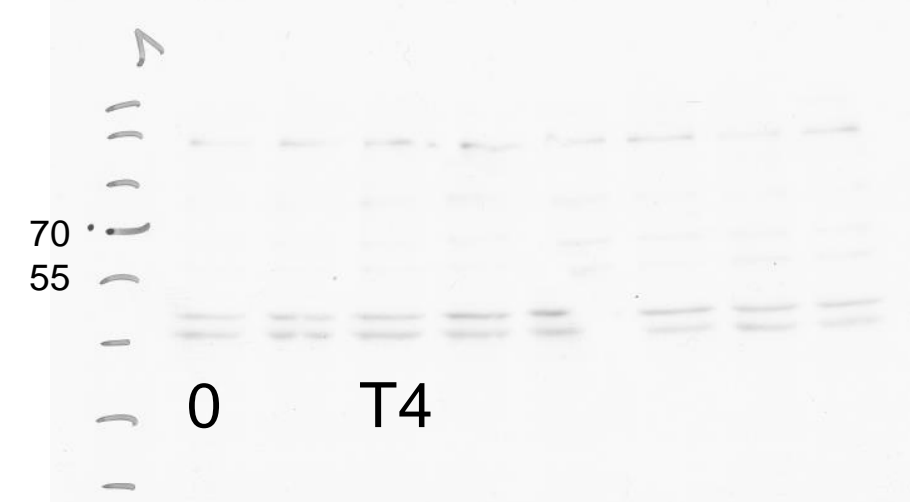

$1 \sec \beta$-Actin

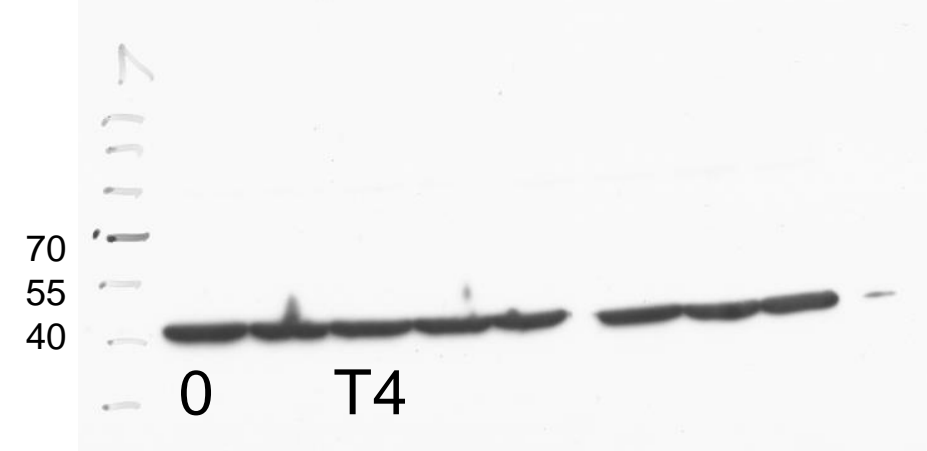

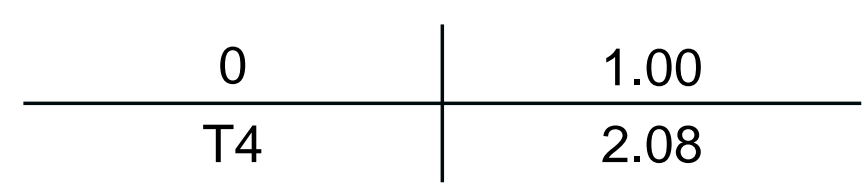

\section{Min CD36}

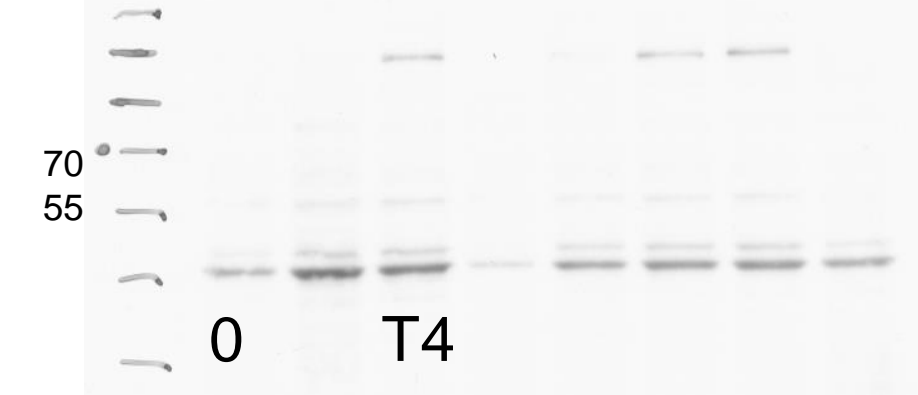

3sec $\beta$-Actin

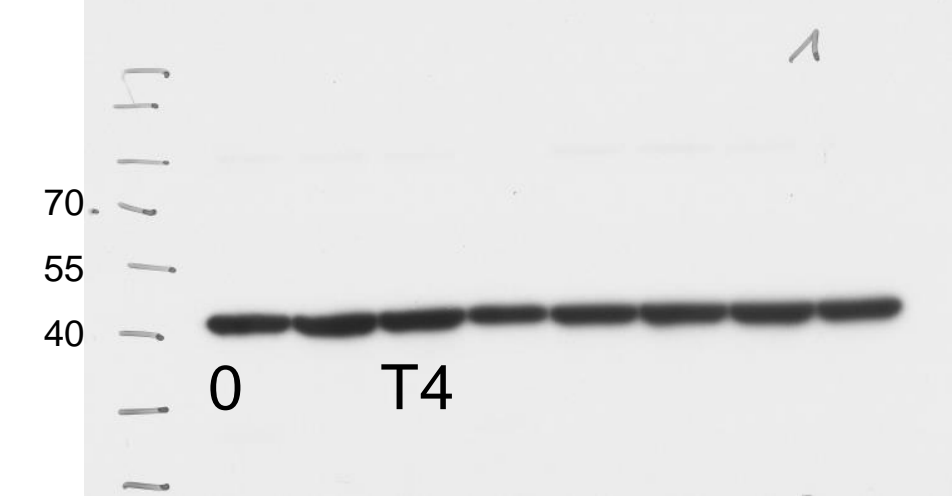

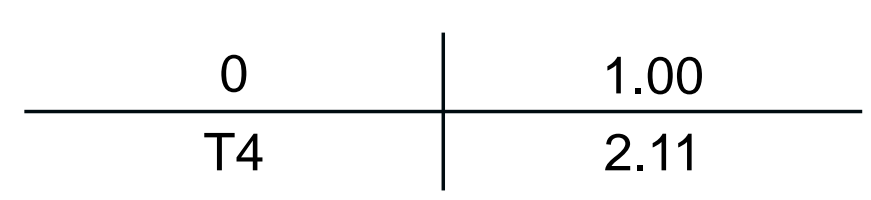

5Min CD36

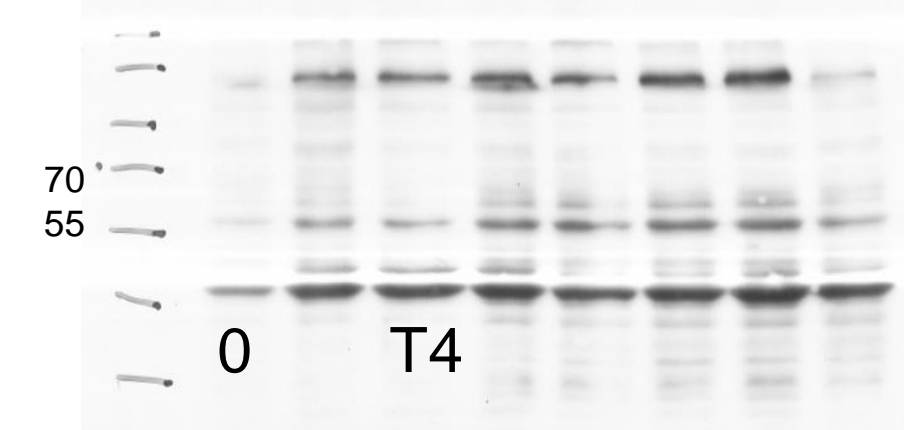

3sec $\beta$-Actin

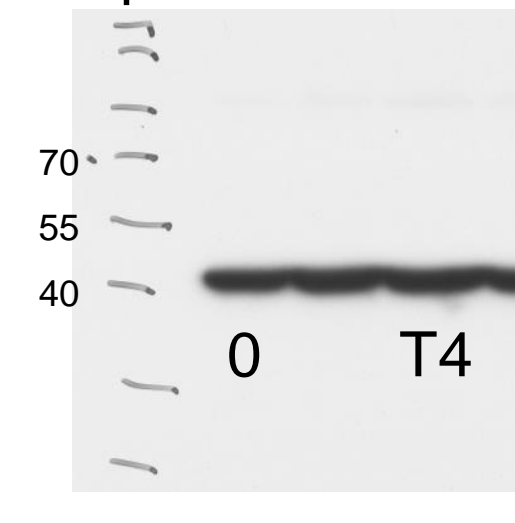


24h after transfection with THR $\alpha / \beta$ targeting siRNA: T4 induced CD36 expression Predicted band size: $53 \mathrm{kDa}, \beta$-Actin $42 \mathrm{kDa}$

\begin{tabular}{c|c}
0 & 1.00 \\
\hline T4 & 1.75
\end{tabular}

1 Min CD36

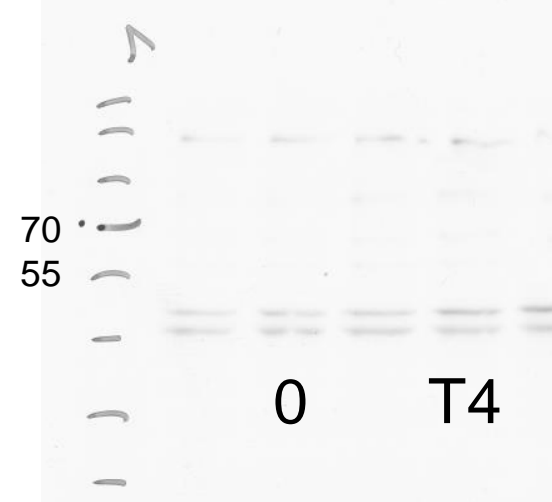

$1 \sec \beta$-Actin

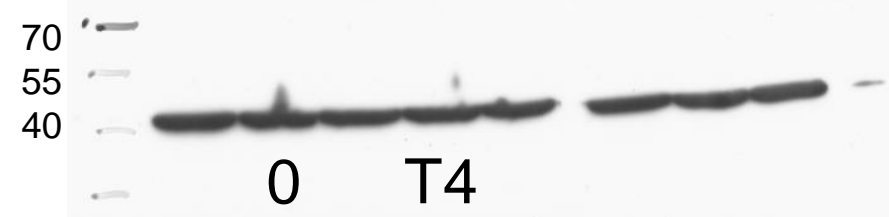

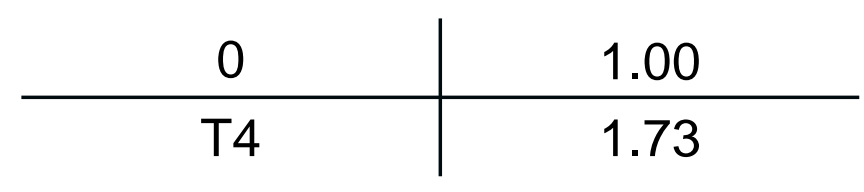

5Min CD36

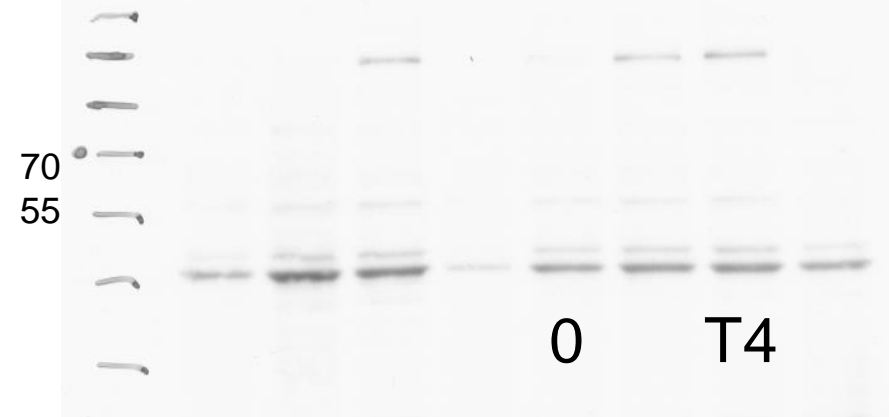

$1 \sec \beta$-Actin

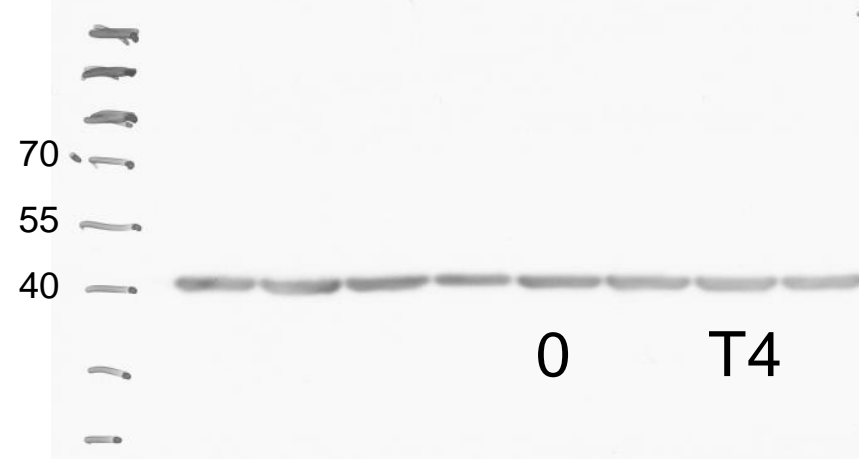

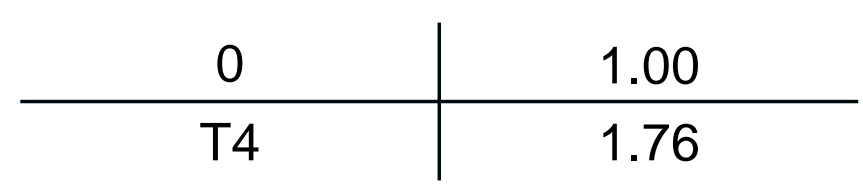

5Min CD36

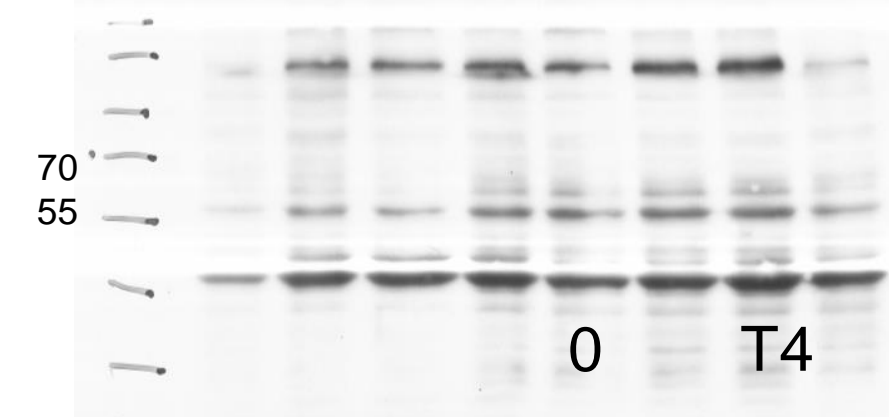

$1 \sec \beta$-Actin

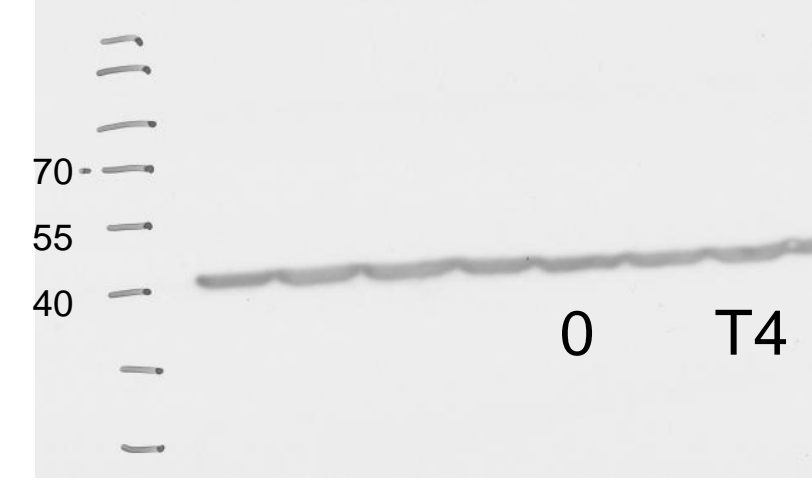


24h after transfection with non-targeting siRNA: TETRAC induced CD36 expression Predicted band size: $53 \mathrm{kDa}, \beta$-Actin $42 \mathrm{kDa}$

\begin{tabular}{c|c}
0 & 1.00 \\
\hline TETRAC & 1.86
\end{tabular}
30 sec CD36

\section{$1 \sec \beta$-Actin}

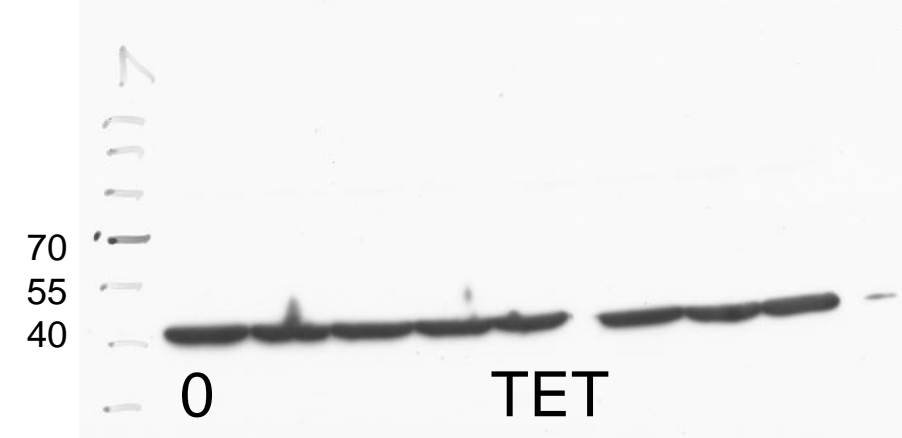

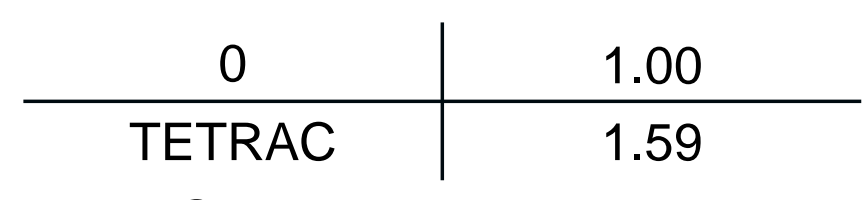

\section{Min CD36}

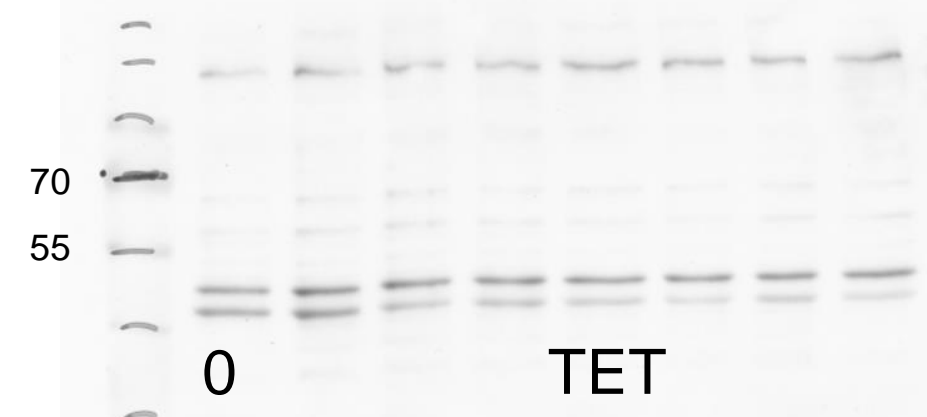

$1 \sec \beta$-Actin

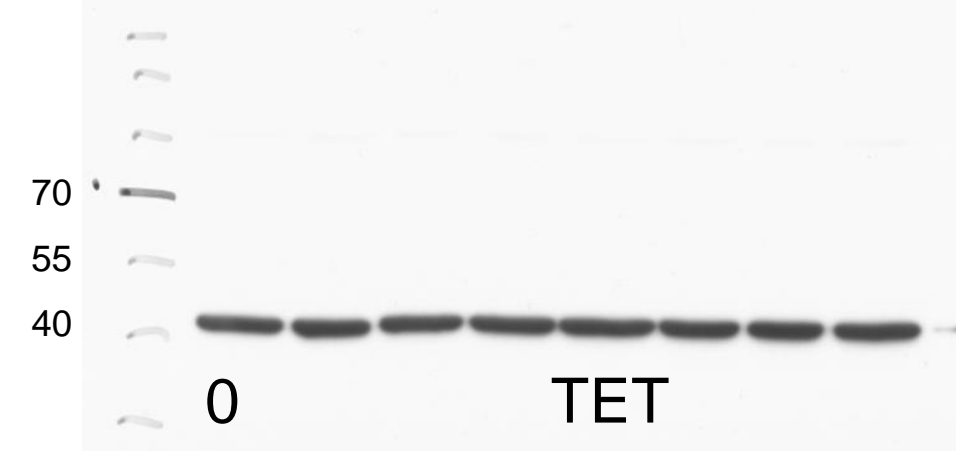

\begin{tabular}{c|c}
0 & 1.00 \\
\hline TETRAC & 1.95
\end{tabular}
5Min CD36

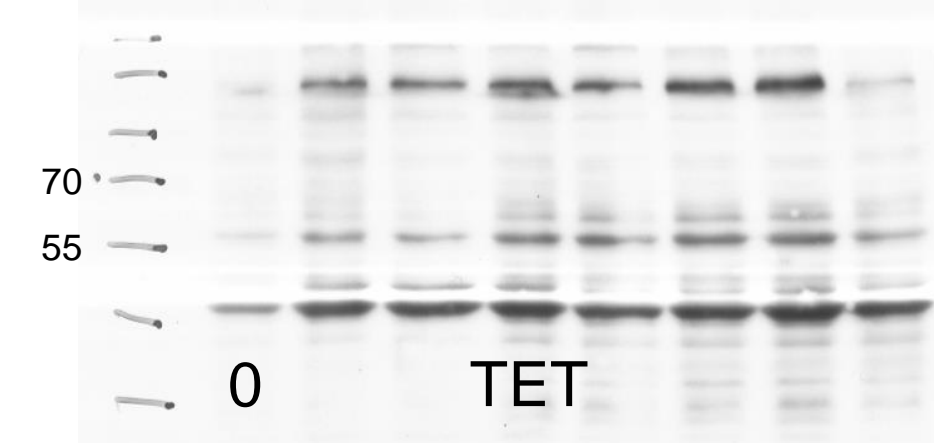

$1 \sec \beta$-Actin

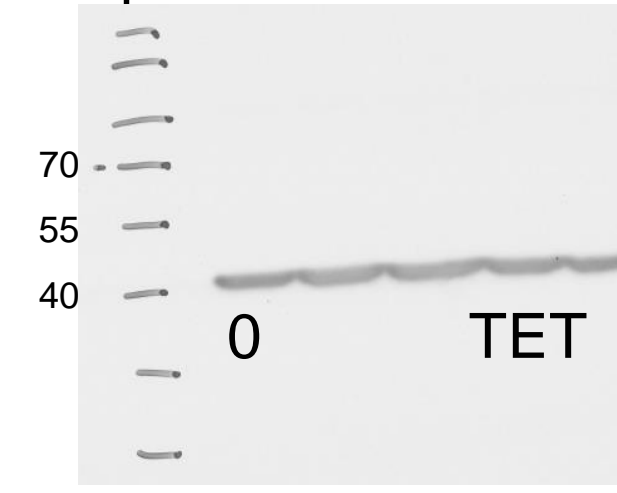


24h after transfection with THR $\alpha / \beta$ targeting siRNA: TETRAC induced CD36 expression

Predicted band size: $53 \mathrm{kDa}, \beta$-Actin $42 \mathrm{kDa}$

\begin{tabular}{c|c}
0 & 1.00 \\
\hline TETRAC & 1.83
\end{tabular}

1 Min CD36

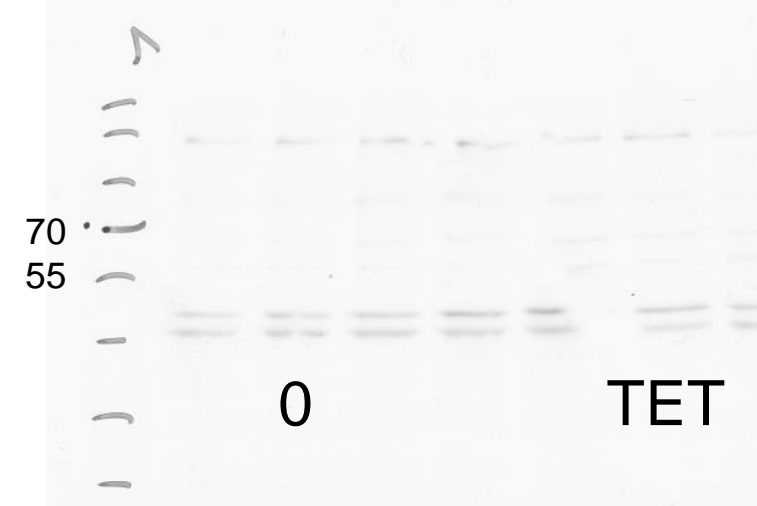

3sec $\beta$-Actin

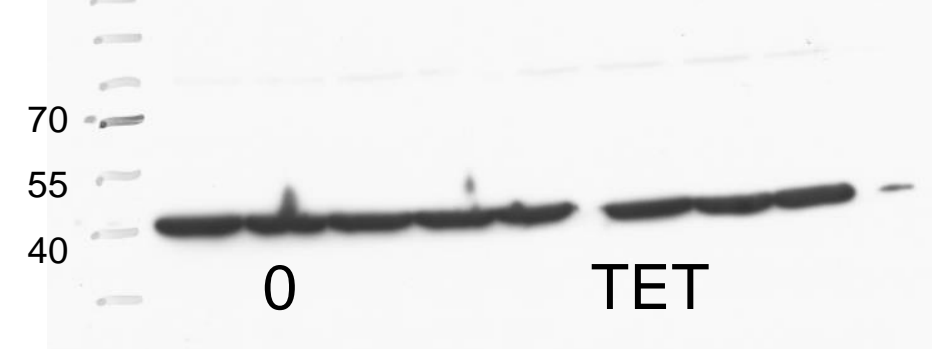

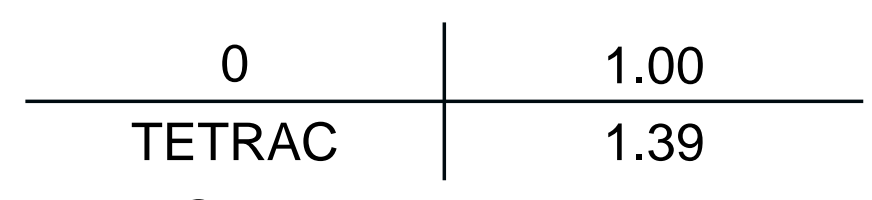

5Min CD36

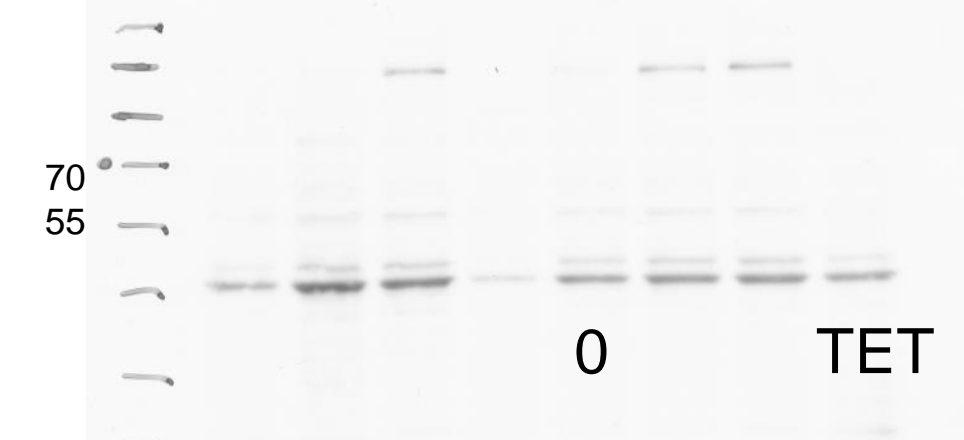

$1 \sec \beta$-Actin

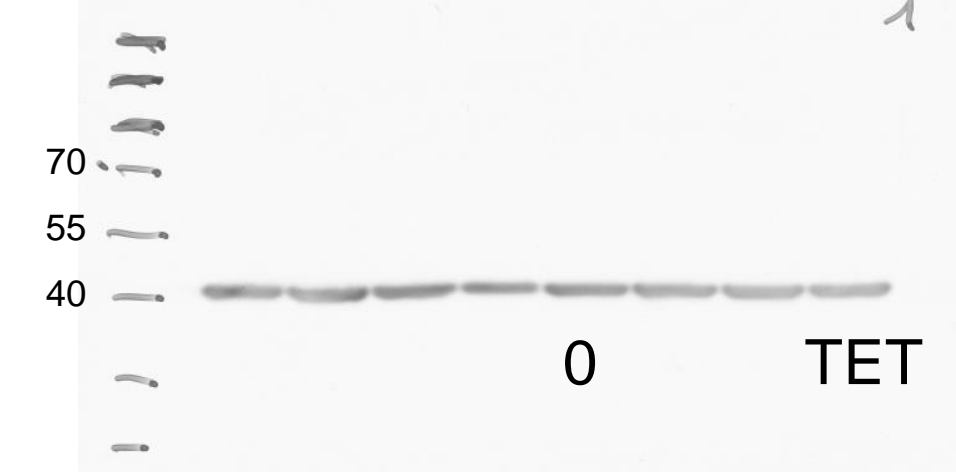

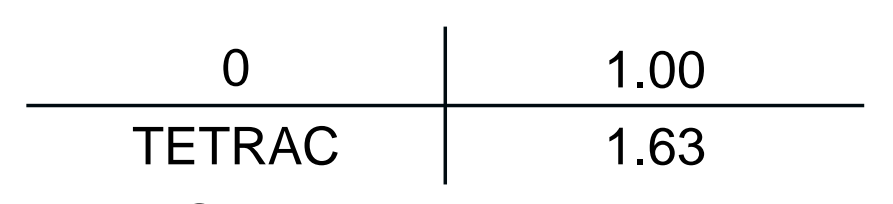

1 Min CD36

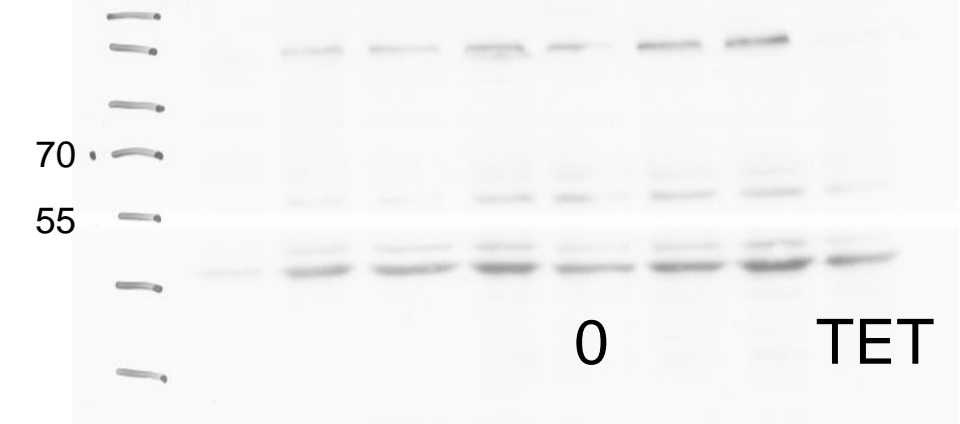

$1 \sec \beta$-Actin

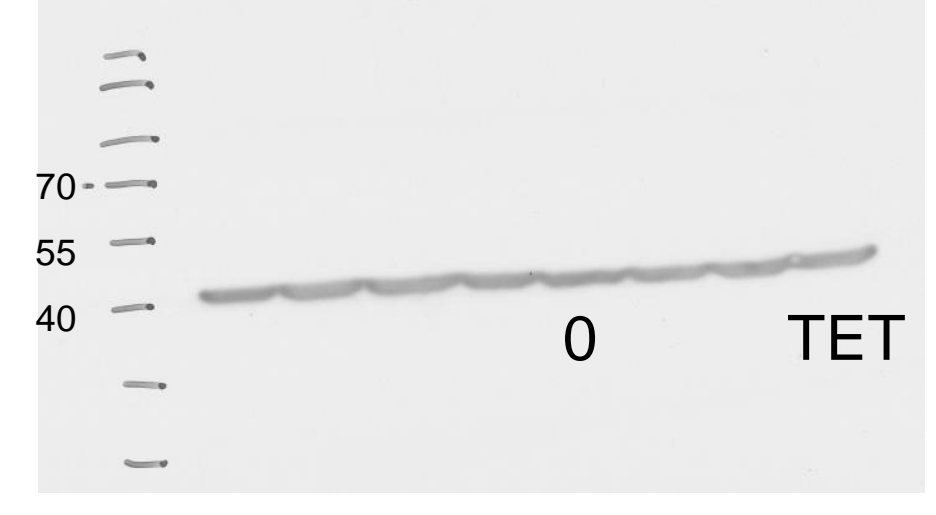


24h after transfection with non-targeting siRNA: Pio induced CD36 expression Predicted band size: $53 \mathrm{kDa}, \beta$-Actin $42 \mathrm{kDa}$

\begin{tabular}{c|c}
0 & 1.00 \\
\hline Pio & 1.98
\end{tabular}

30sec CD36

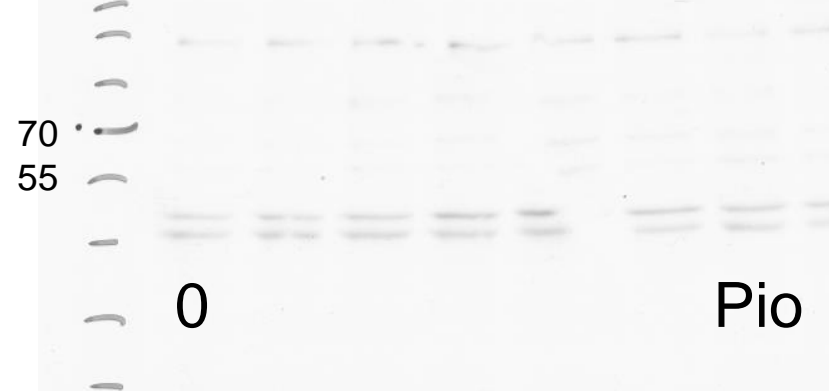

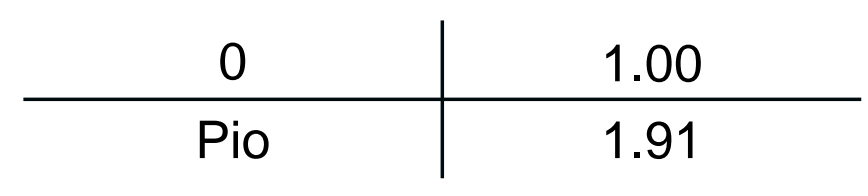

5Min CD36

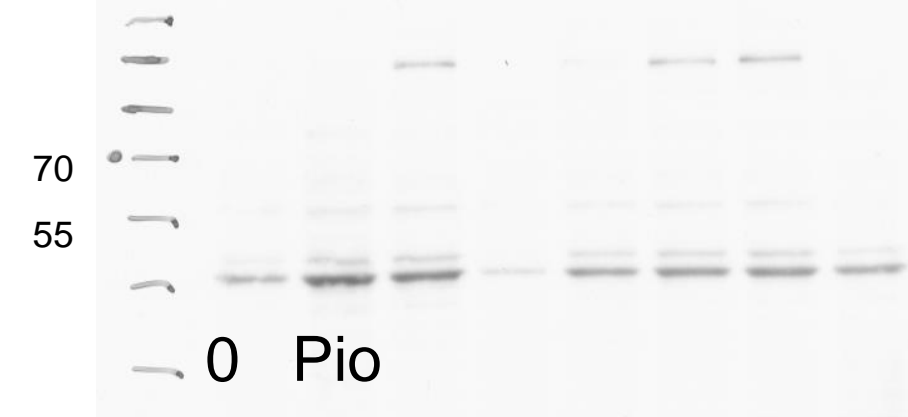

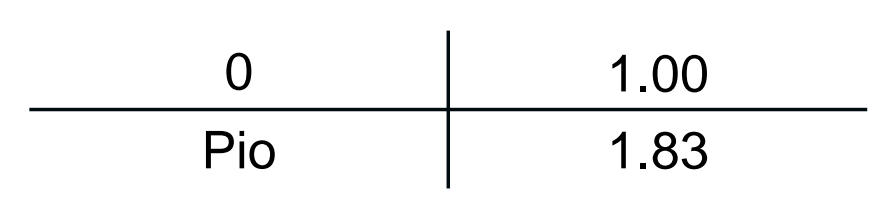

5Min CD36

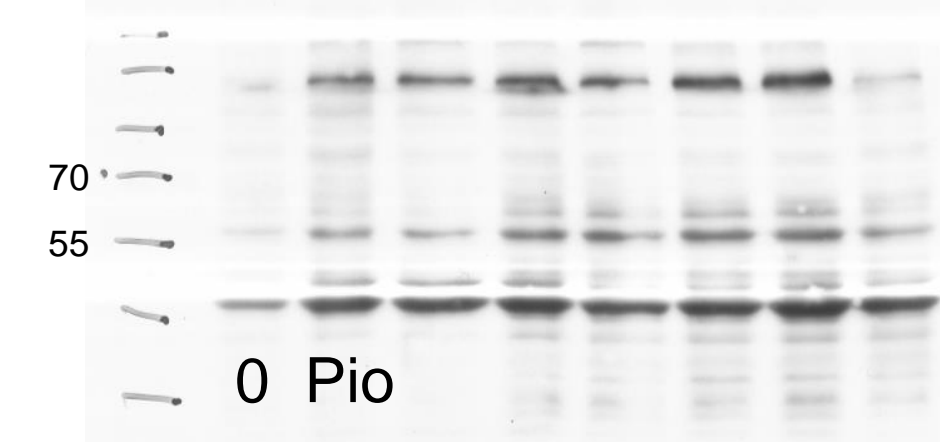

\section{3sec $\beta$-Actin}

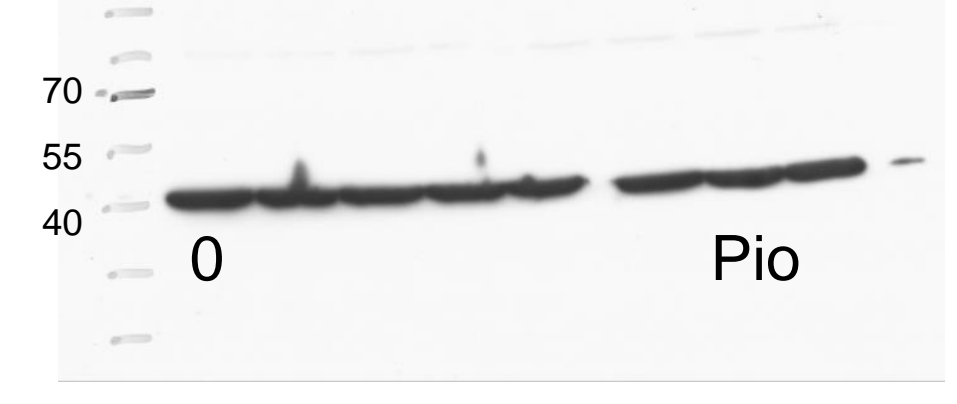

$1 \sec \beta$-Actin

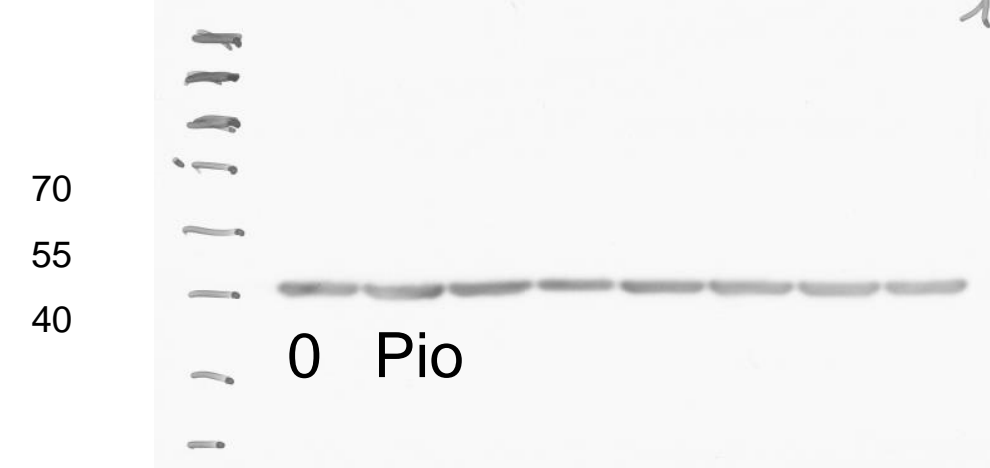

\section{$1 \sec \beta$-Actin}

$$
\begin{aligned}
70 & = \\
55 & = \\
40 & = \\
& -0 \text { Pio }
\end{aligned}
$$


24h after transfection with THR $\alpha / \beta$ targeting siRNA: Pio induced CD36 expression Predicted band size: $53 \mathrm{kDa}, \beta$-Actin $42 \mathrm{kDa}$

\begin{tabular}{c|c}
0 & 1.00 \\
\hline Pio & 1.96
\end{tabular}

1 Min CD36

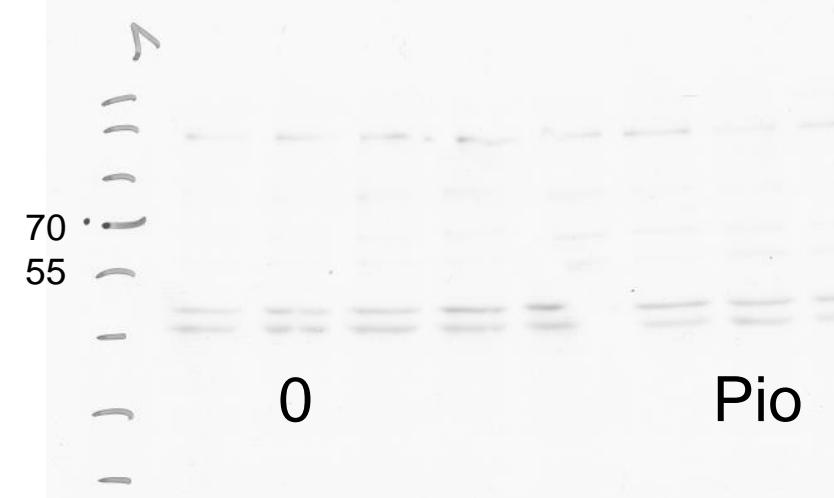

3sec $\beta$-Actin

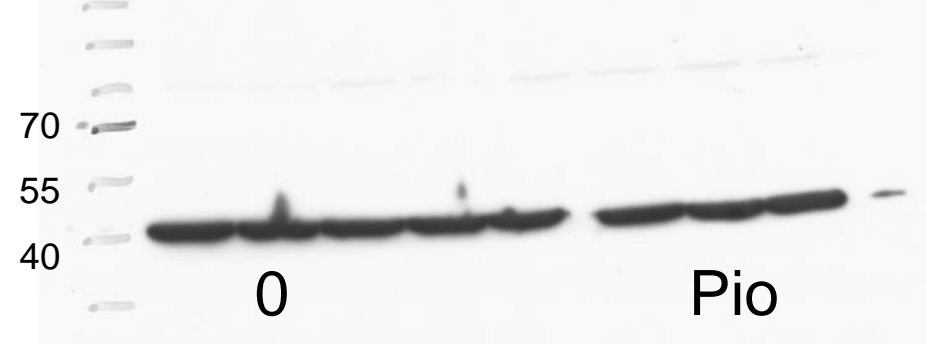

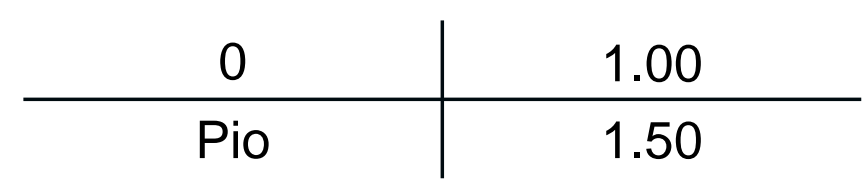

\section{Min CD36}

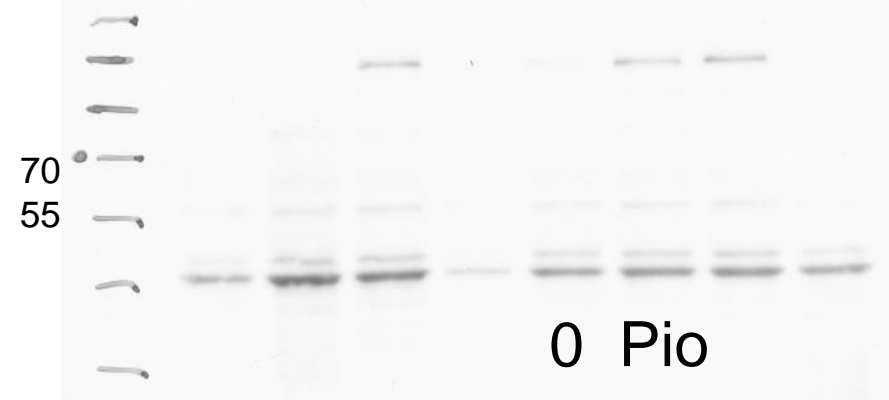

$1 \sec \beta$-Actin

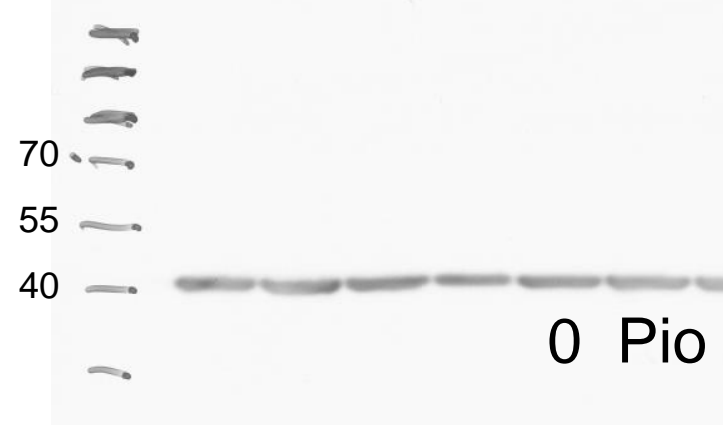

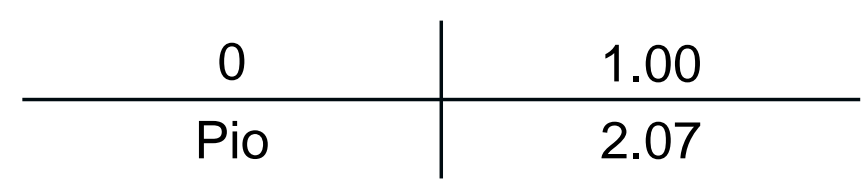

1 Min CD36

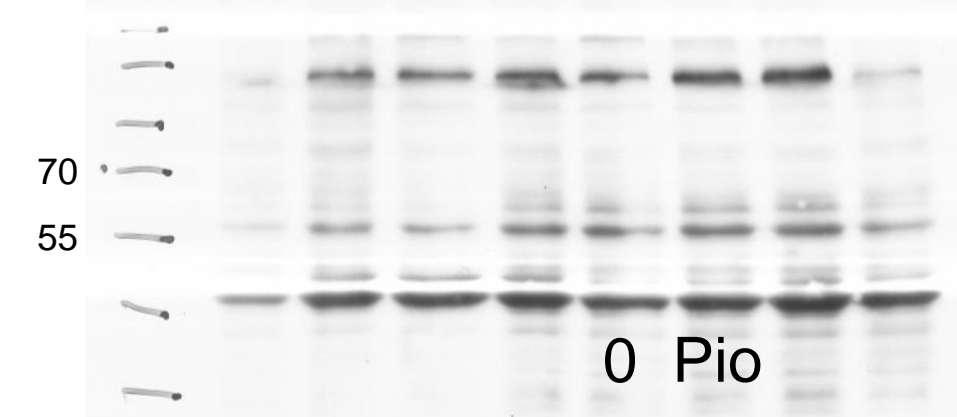

$1 \sec \beta$-Actin

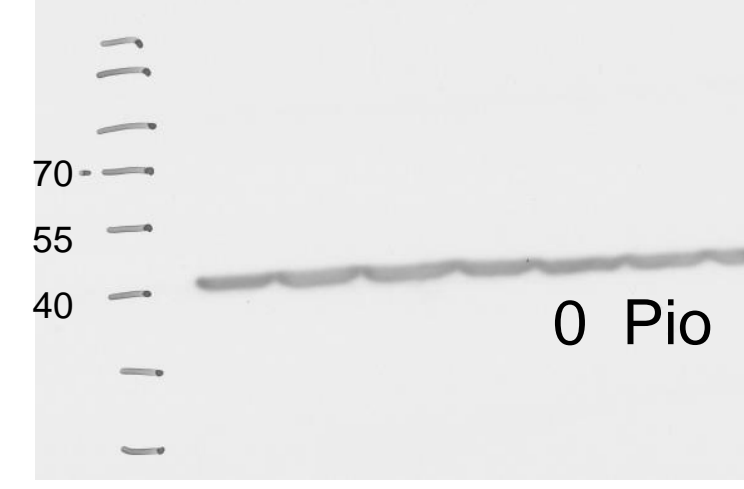


48h after transfection with non-targeting siRNA: T4 induced CD36 expression

Predicted band size: $53 \mathrm{kDa}, \beta$-Actin $42 \mathrm{kDa}$

\begin{tabular}{c|c}
0 & 1.00 \\
\hline T4 & 2.03
\end{tabular}

5Min CD36

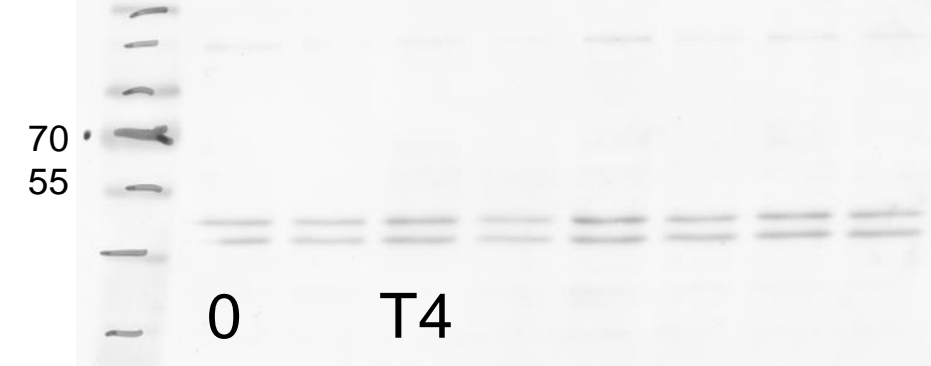

$1 \sec \beta$-Actin

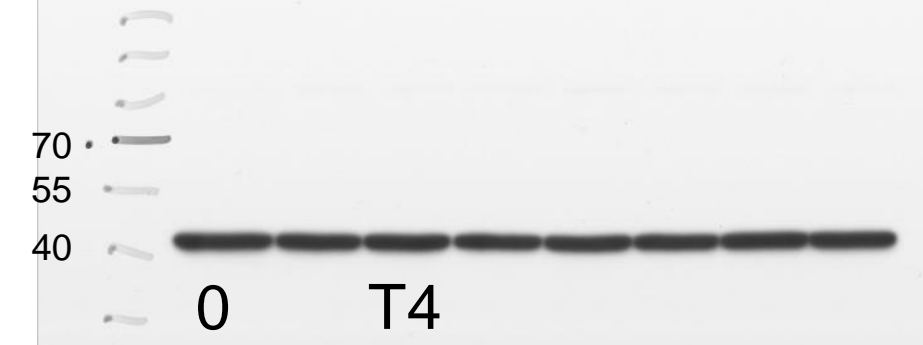

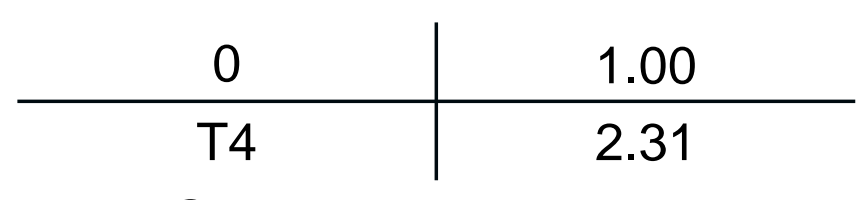

5Min CD36

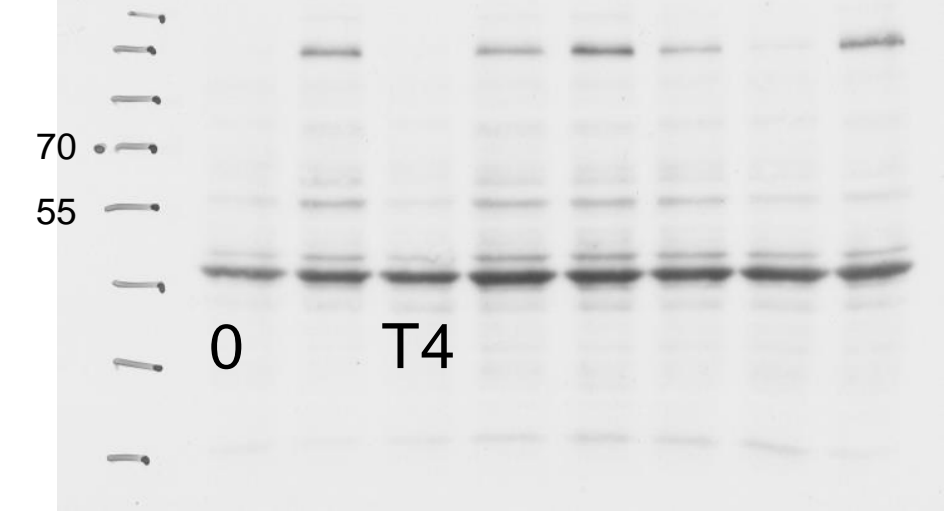

$1 \sec \beta$-Actin

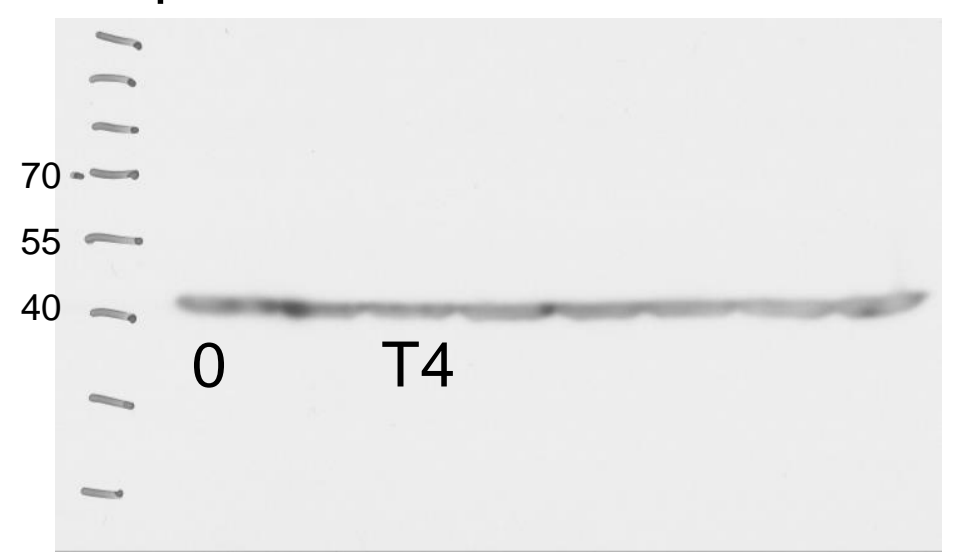

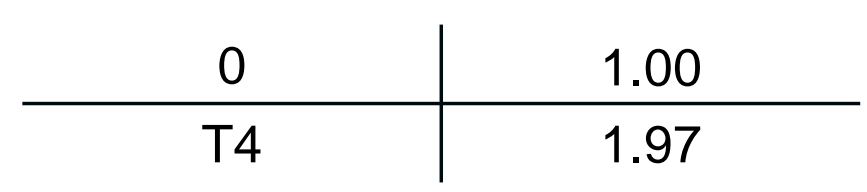

\section{Min CD36}

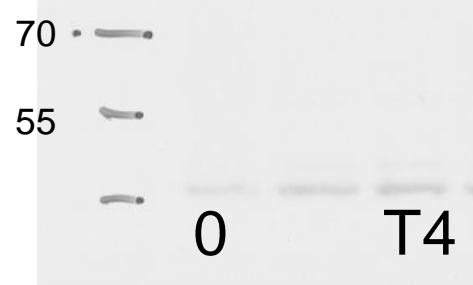

\section{3sec $\beta$-Actin}

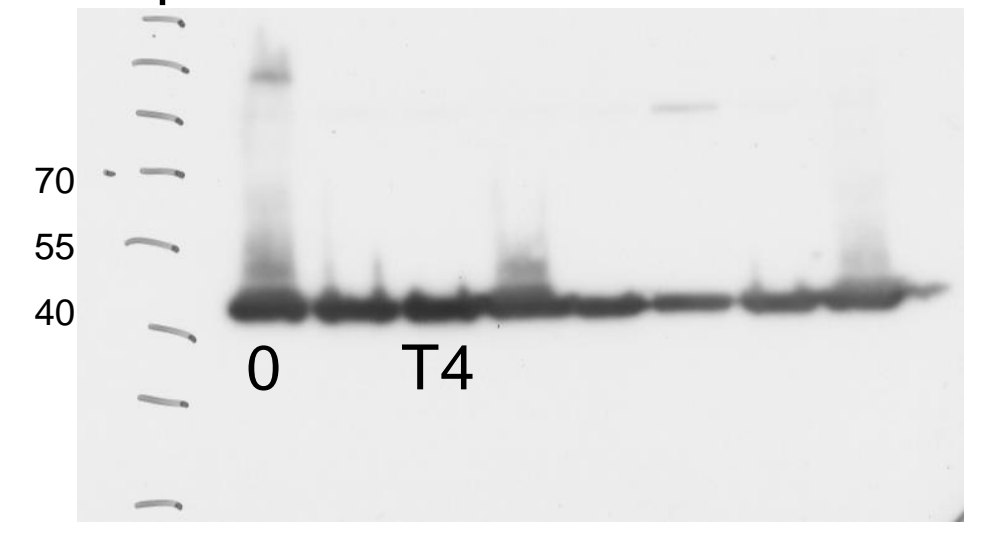


48h after transfection with THR $\alpha / \beta$ targeting siRNA: T4 induced CD36 expression

Predicted band size: $53 \mathrm{kDa}, \beta$-Actin $42 \mathrm{kDa}$

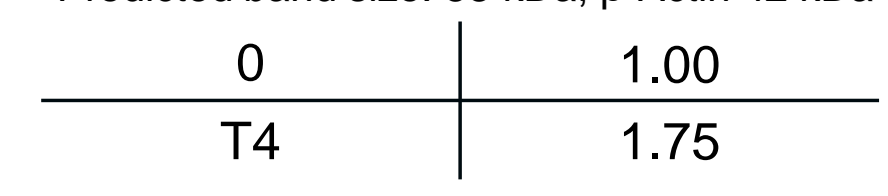

5Min CD36

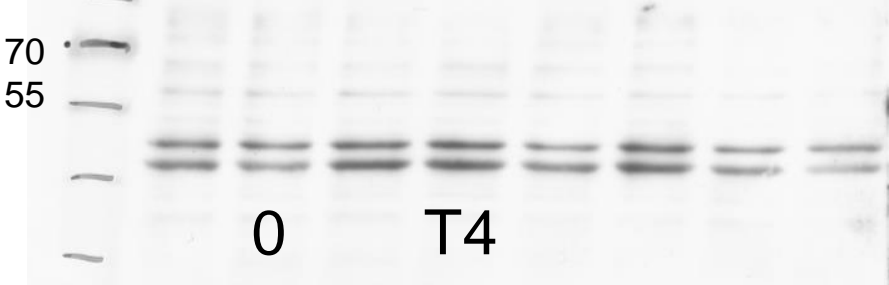

\section{3sec $\beta$-Actin}$$
70
$$$$
55
$$$$
40
$$$$
0 \quad \mathrm{~T} 4
$$

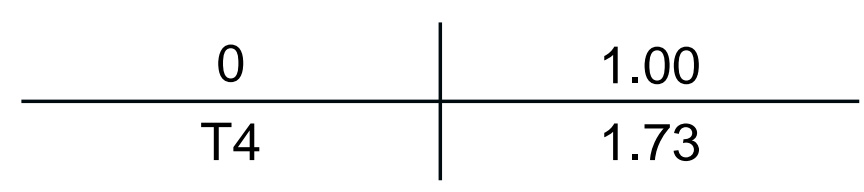

1 Min CD36

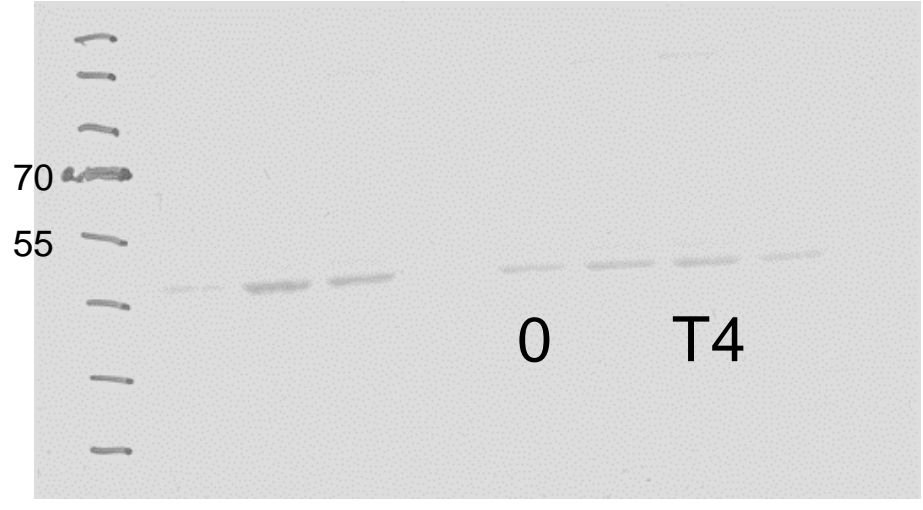

$1 \sec \beta$-Actin

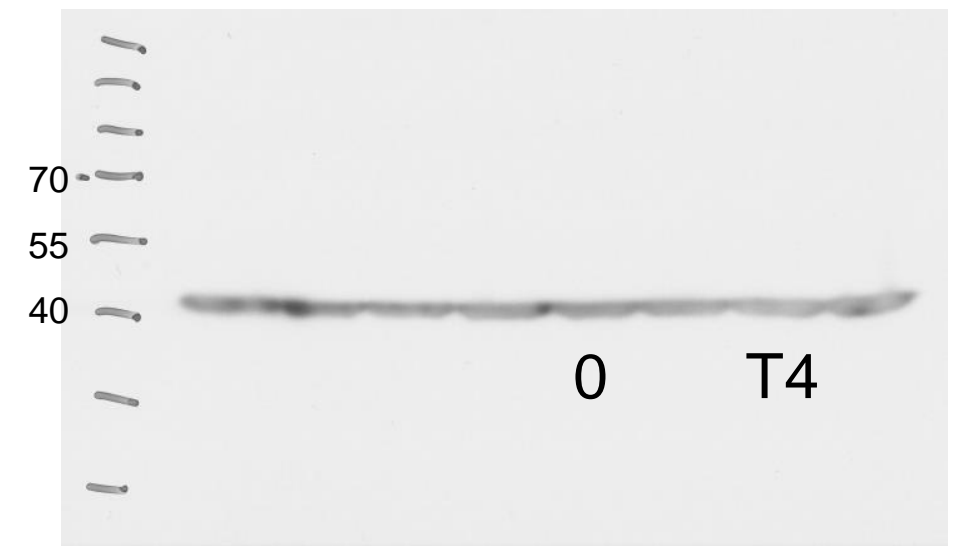

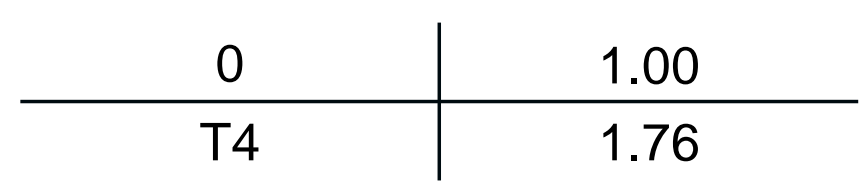

5Min CD36

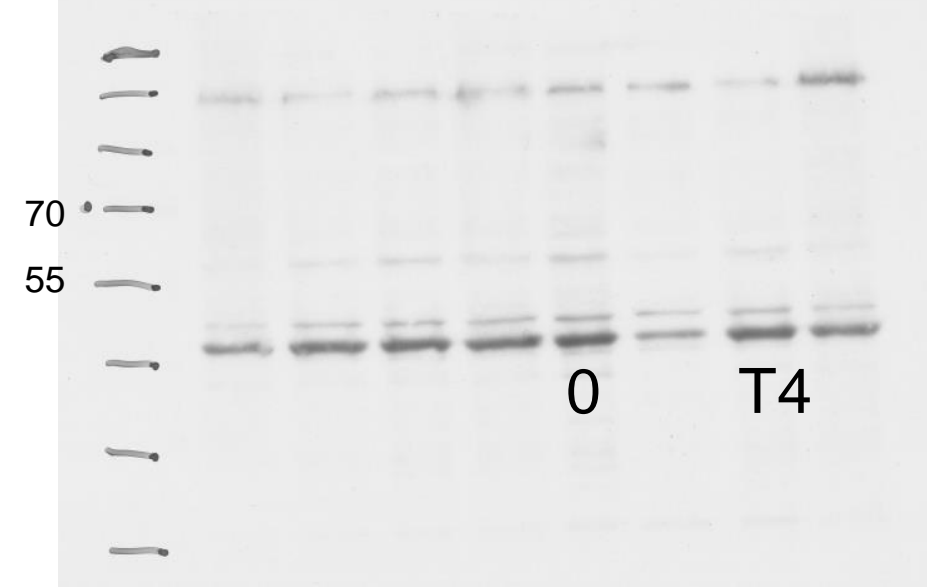

$1 \sec \beta$-Actin

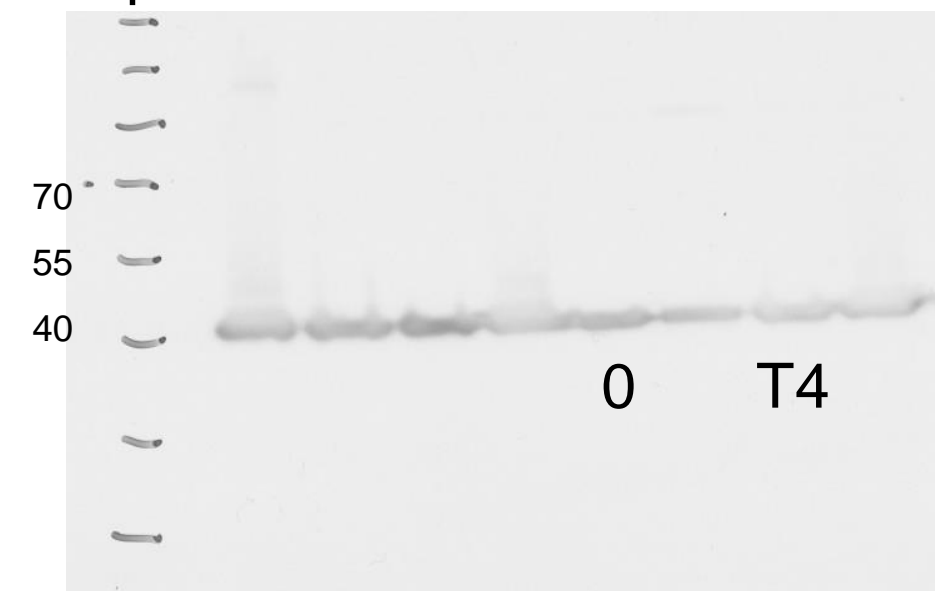


48h after transfection with non-targeting siRNA: TETRAC induced CD36 expression

Predicted band size: $53 \mathrm{kDa}, \beta$-Actin $42 \mathrm{kDa}$
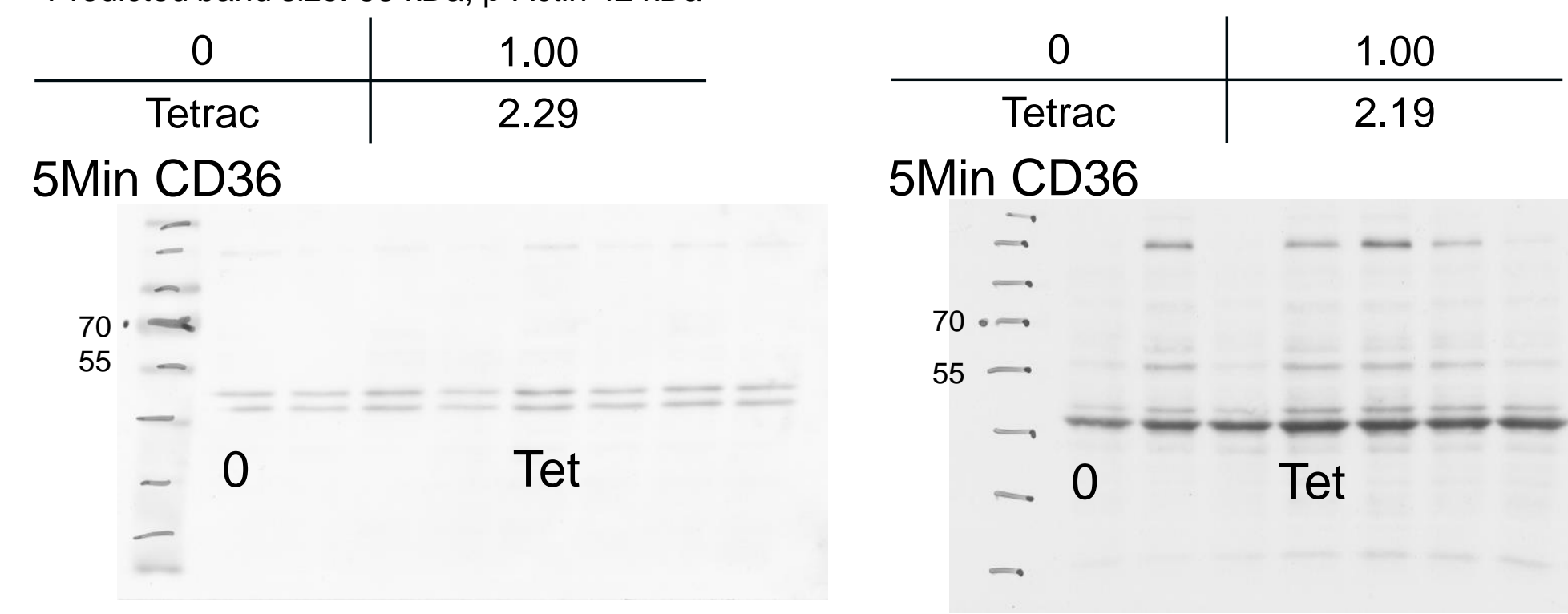

\section{Min CD36}

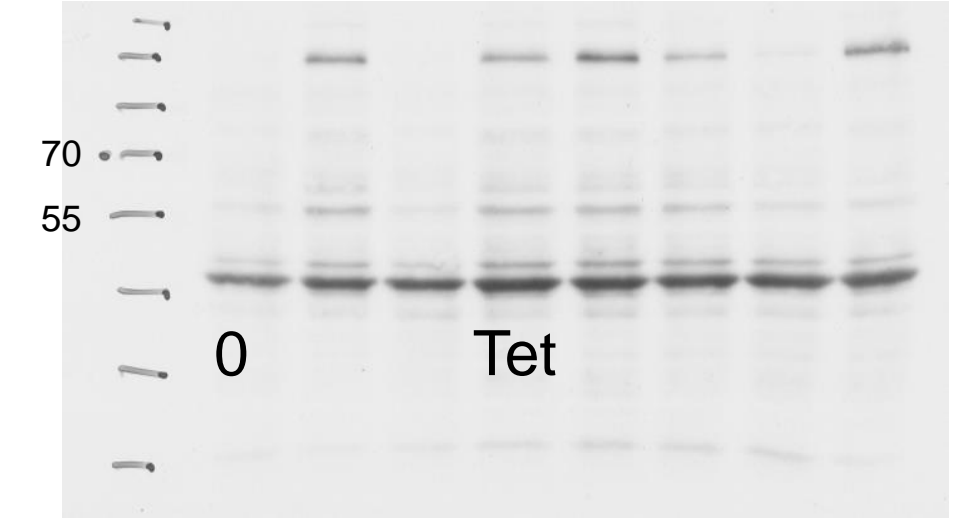

1 sec $\beta$-Actin

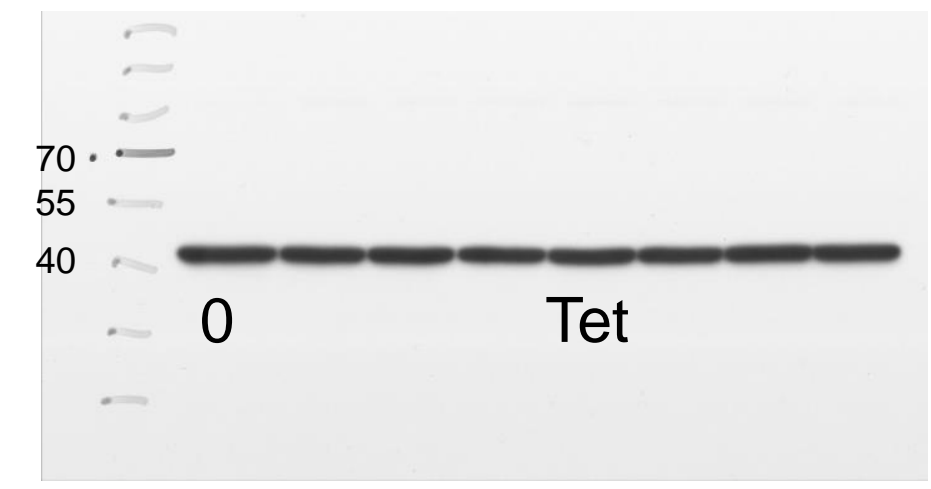

1sec $\beta$-Actin

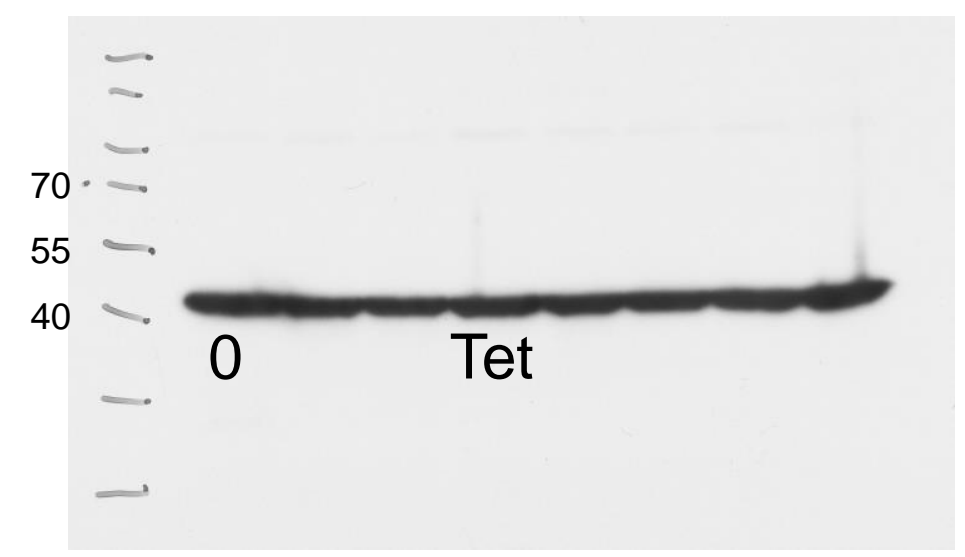

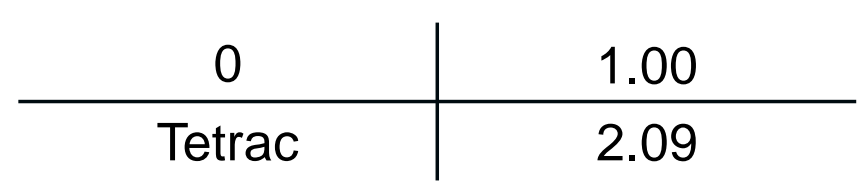

5Min CD36

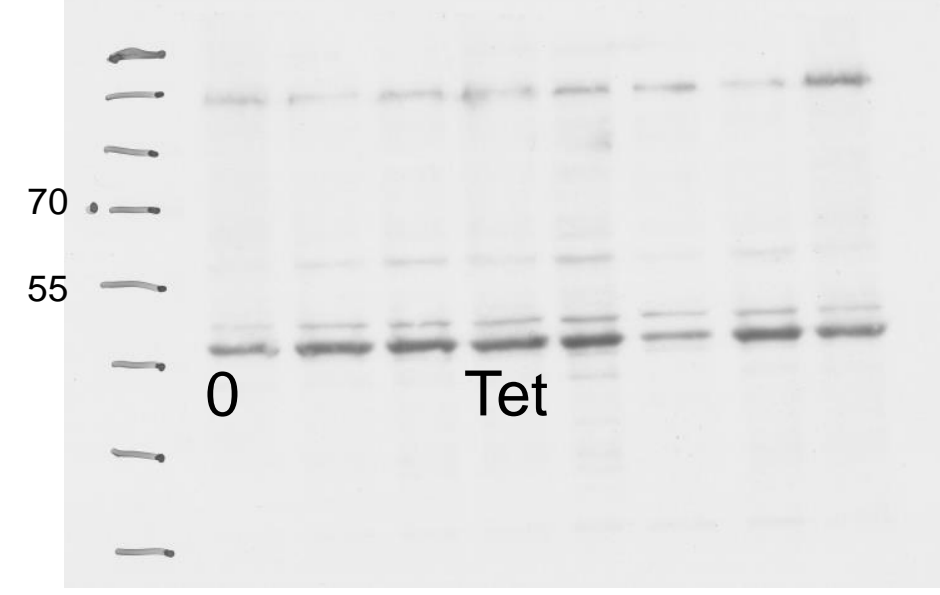

1sec $\beta$-Actin

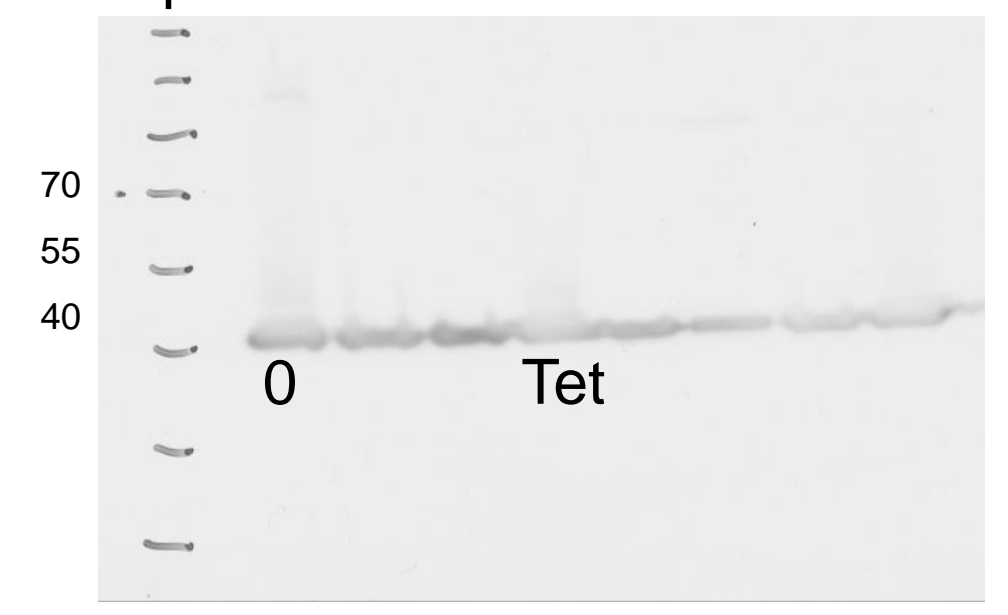


48h after transfection with THR $\alpha / \beta$ targeting siRNA: TETRAC induced CD36 expression

Predicted band size: $53 \mathrm{kDa}, \beta$-Actin $42 \mathrm{kDa}$

\begin{tabular}{c|c}
0 & 1.00 \\
\hline Tetrac & 1.96
\end{tabular}

5Min CD36

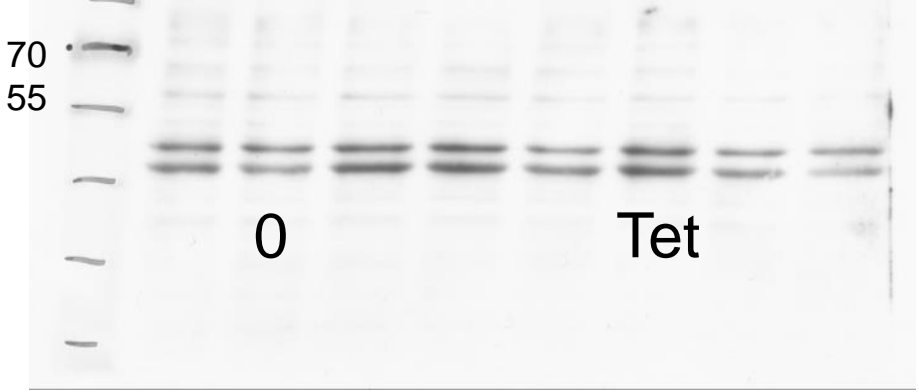

3sec $\beta$-Actin

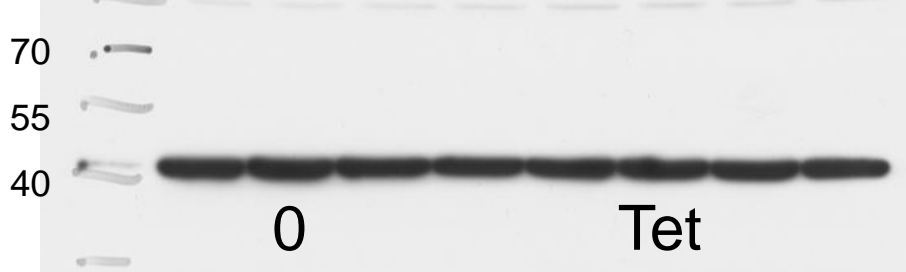

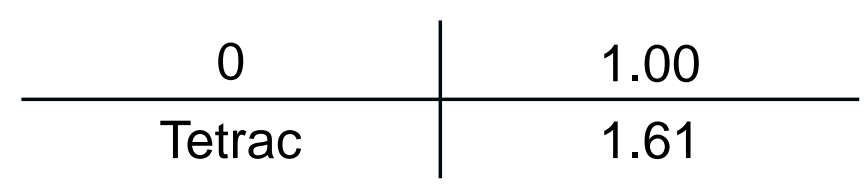

5Min CD36

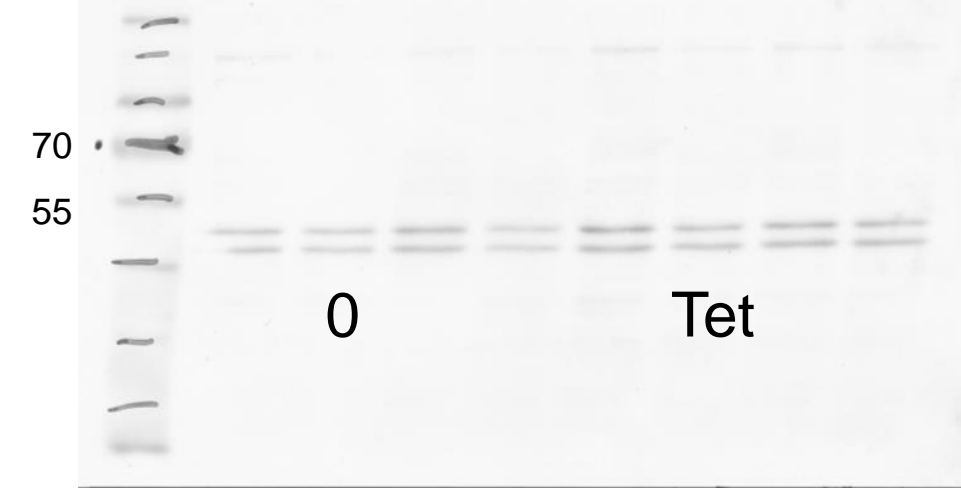

3sec $\beta$-Actin

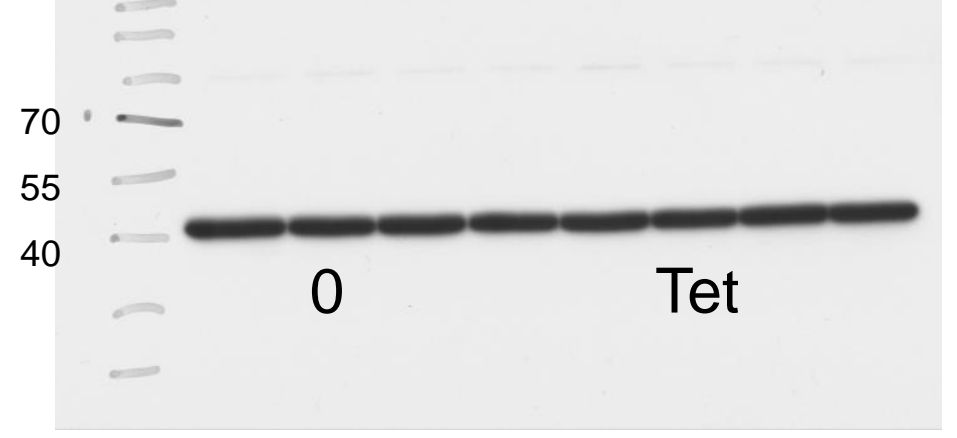

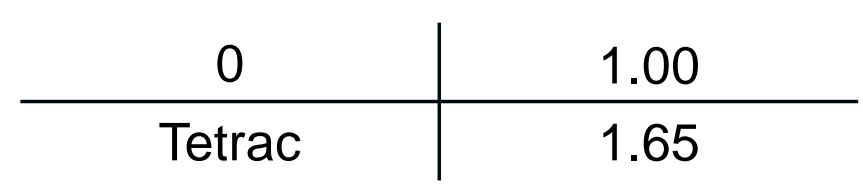

5Min CD36

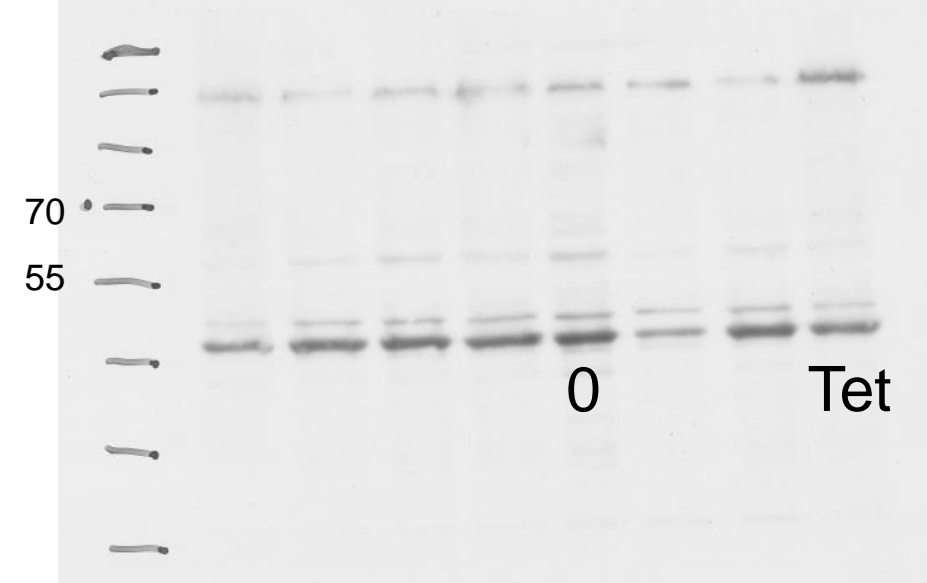

$1 \sec \beta$-Actin

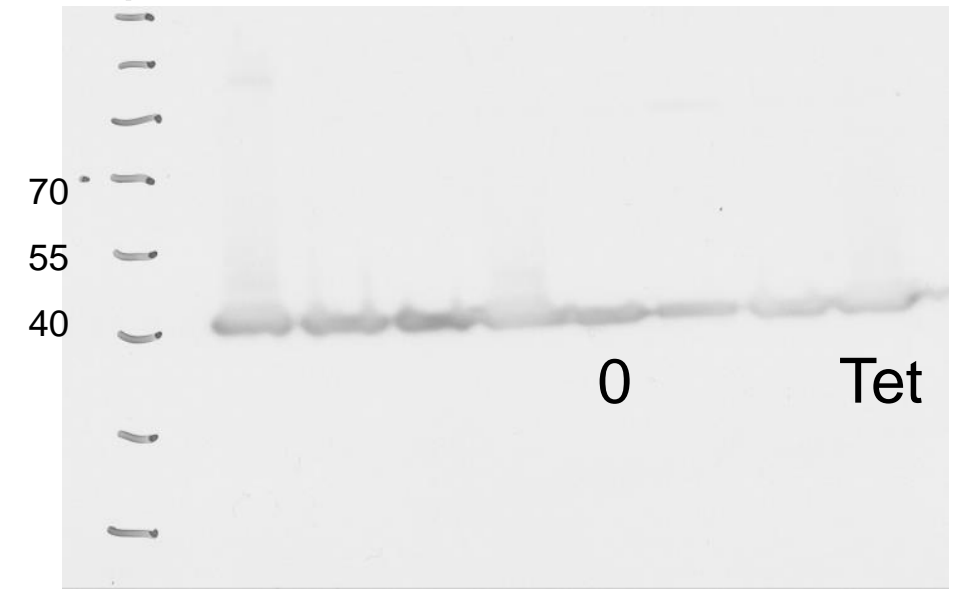


48h after transfection with non-targeting siRNA: Pio induced CD36 expression

Predicted band size: $53 \mathrm{kDa}, \beta$-Actin $42 \mathrm{kDa}$

\begin{tabular}{c|c}
0 & 1.00 \\
\hline Pio & 2.05
\end{tabular}

$5 \min$ CD36

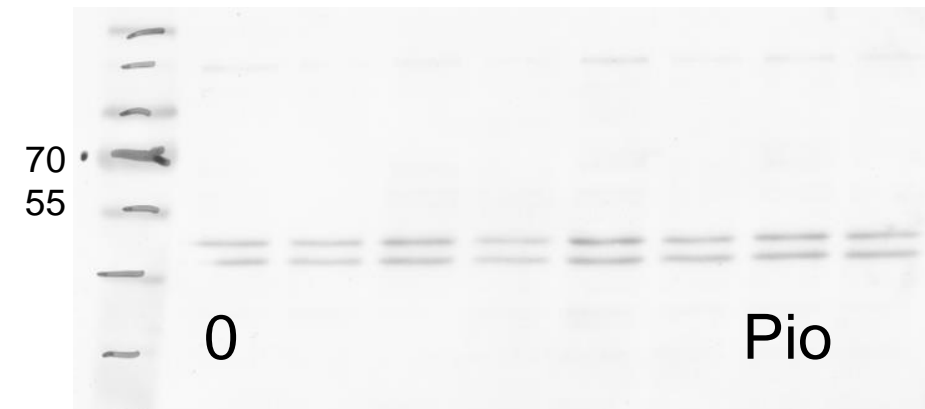

$1 \sec \beta$-Actin

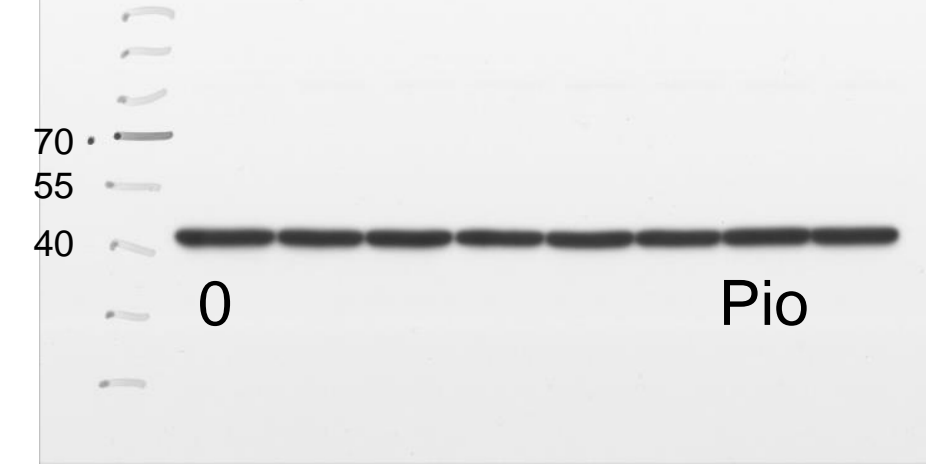

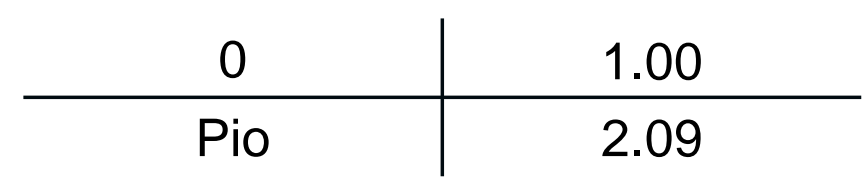

$30 \sec$ CD36

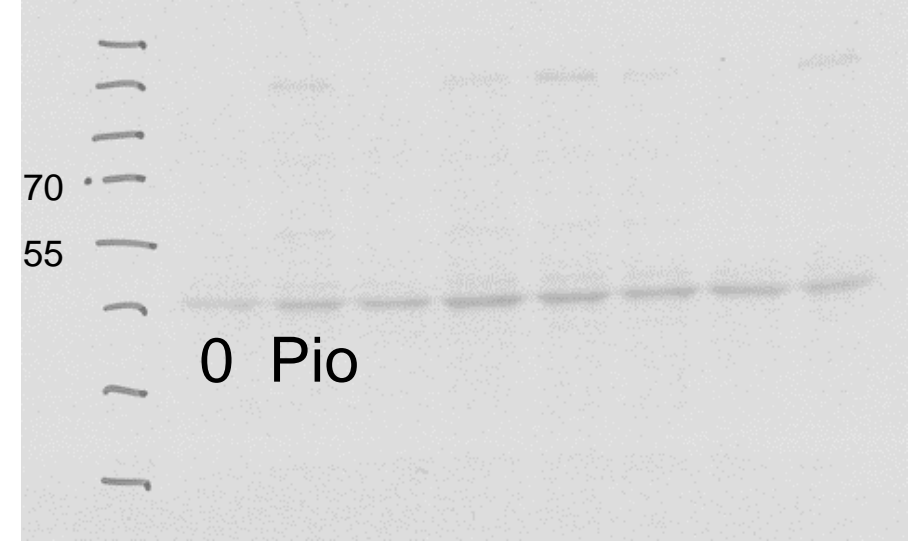

$1 \sec \beta$-Actin

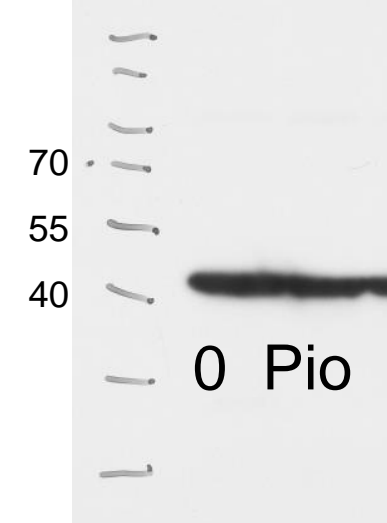

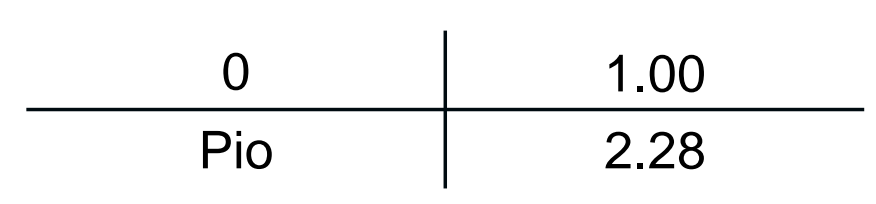

$5 \min$ CD36

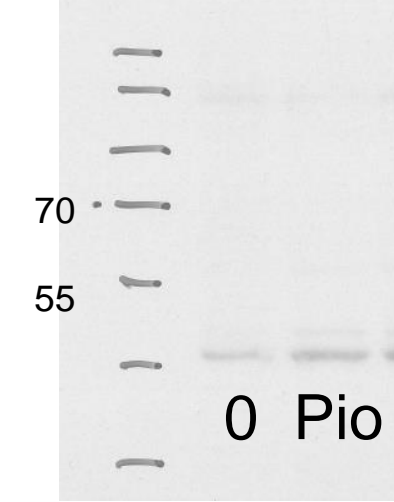

$1 \sec \beta$-Actin

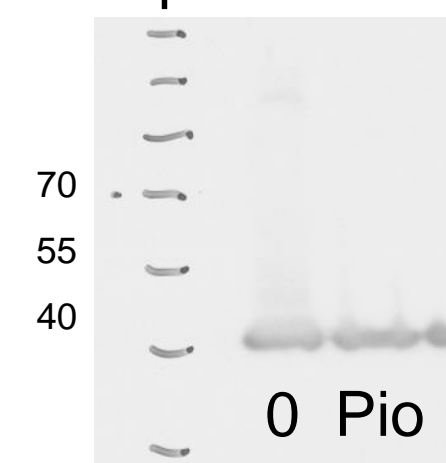


48h after transfection with THRa/ $\beta$ targeting siRNA: Pio induced CD36 expression

Predicted band size: $53 \mathrm{kDa}, \beta$-Actin $42 \mathrm{kDa}$

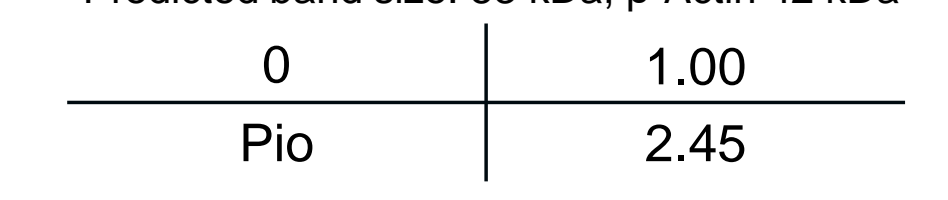

5Min CD36

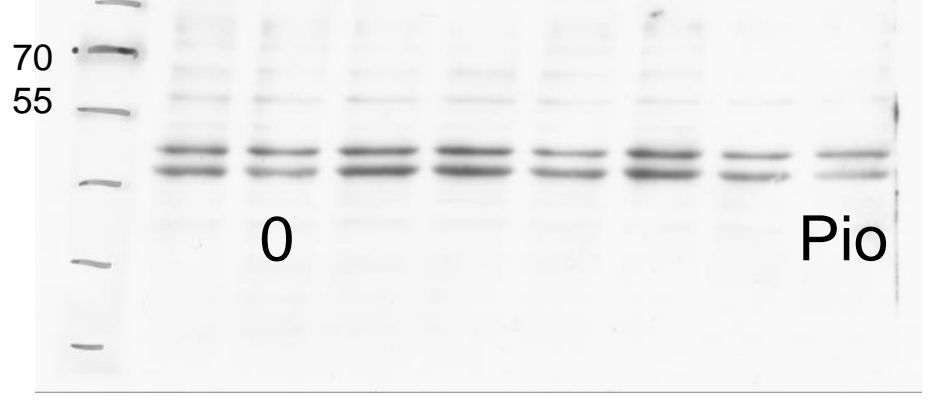

3sec $\beta$-Actin

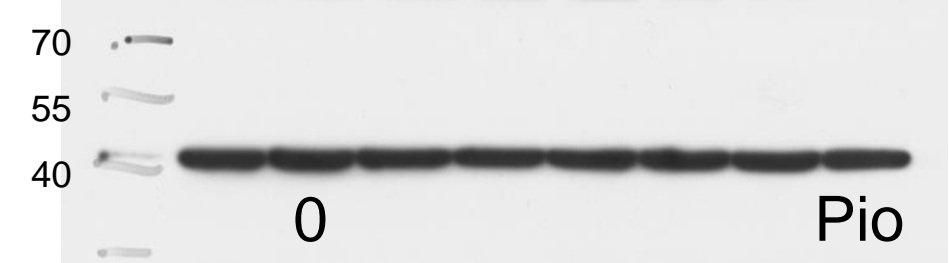

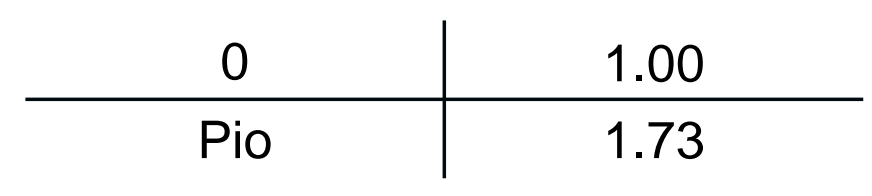

5Min CD36

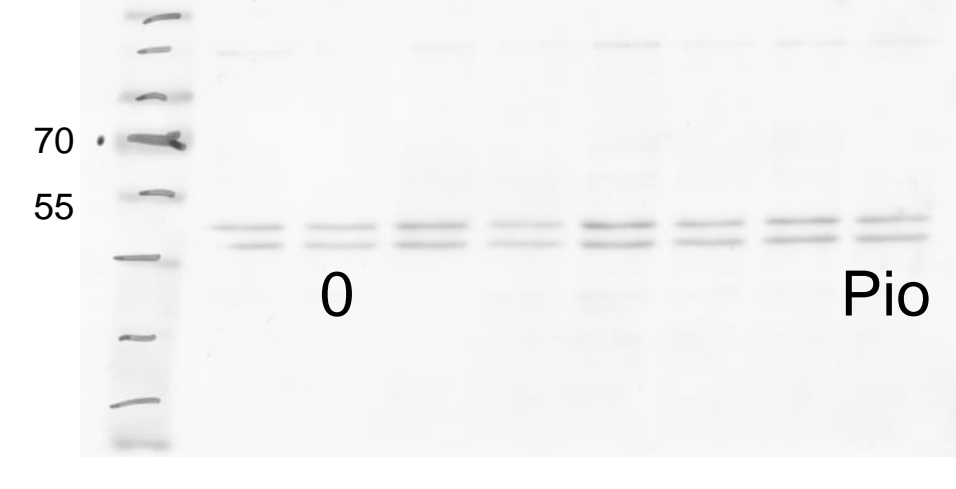

$1 \sec \beta$-Actin

0

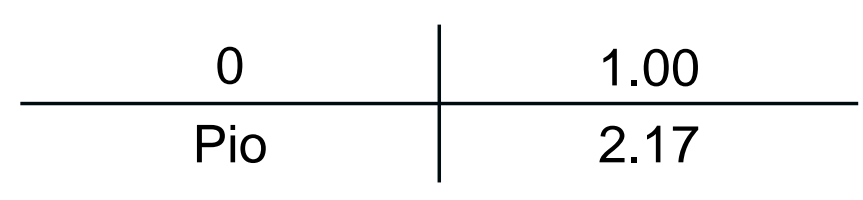

5Min CD36

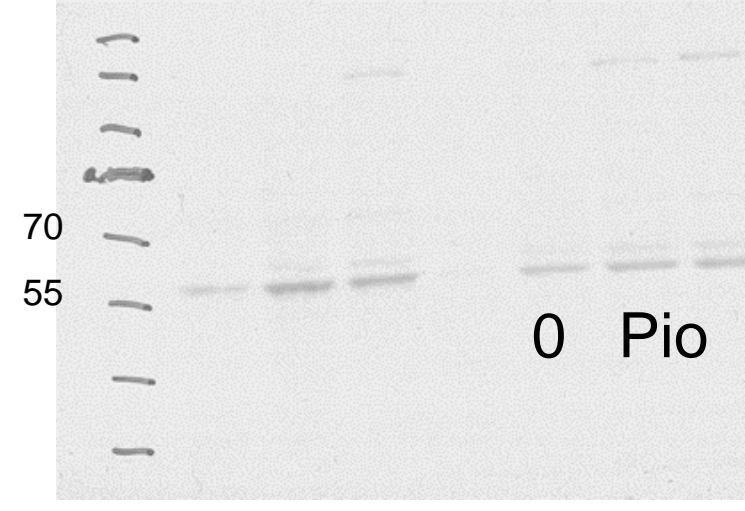

$1 \sec \beta$-Actin

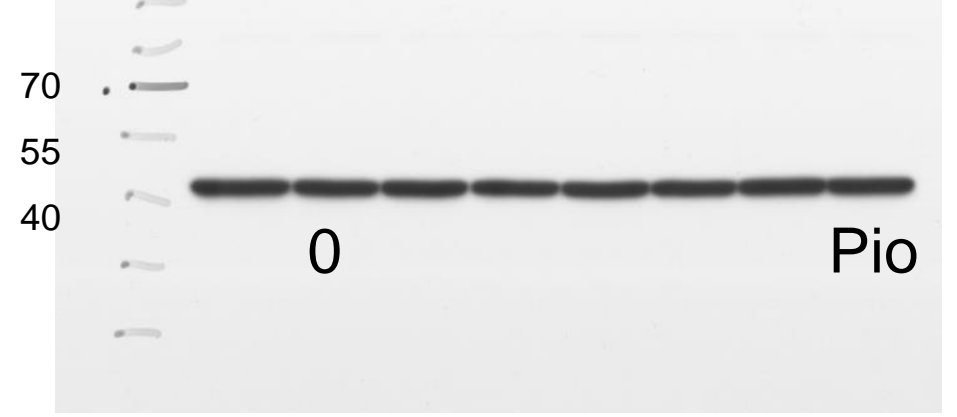

0 Pio 